\title{
Cone Bioassays Provide Reproducible Bioefficacy Estimates with Different Anopheline Mosquitoes and Can Be Used for Quality Assurance of Pyrethroid Insecticide Treated Nets
}

\author{
Stephen Gabriel Mbwambo ( $\square$ smbwambo@ihi.or.tz ) \\ Ifakara Health Institute \\ Nakei Bubun \\ Papua New Guinea Institute of Medical Research \\ Emmanuel Mbuba \\ Ifakara Health Institute \\ Jason Moore \\ Ifakara Health Institute \\ Kasiani Mbina \\ Ifakara Health Institute \\ Dismas Kamande \\ Ifakara Health Institute \\ Moses Laman \\ Papua New Guinea Institute of Medical Research \\ Emmanuel Mpolya \\ Nelson Mandela African Institute of Science and Technology \\ Olukayode Odufuwa \\ Ifakara Health Institute \\ Tim Freeman \\ Rotarians Against Malaria \\ Stephan Karl \\ Papua New Guinea Institute of Medical Research \\ Sarah J Moore \\ Ifakara Health Institute https://orcid.org/0000-0002-0938-6654
}

\section{Research Article}

Keywords: Bioefficacy, bioassay, cone bioassay, tunnel test, insecticide treated nets, ITN, long lasting insecticidal nets, LLIN, pyrethroid, mosquito, Anopheles, malaria, quality assurance

Posted Date: January 24th, 2022

DOI: https://doi.org/10.21203/rs.3.rs-1269567/v1

License: (c) (i) This work is licensed under a Creative Commons Attribution 4.0 International License. Read Full License 


\section{Abstract Background}

Quality assurance (QA) of insecticide-treated nets (ITNs) delivered to malaria-endemic countries is conducted by measuring physiochemical parameters, but not bioefficacy against malaria mosquitoes. The cone bioassay provides a simple evaluation of ITN bioefficacy and its conditions and parameters are prescribed by the World Health Organization (WHO). This study explored utility of cone bioassays for predelivery QA of pyrethroid ITNs in two test facilities using different mosquito species to test the assumption that cone bioassays are consistent and reproducible across locations, mosquito strains, and laboratories.

\section{Methods}

Double-blinded bioassays were conducted on unused pyrethroid ITNs of 4 brands ( 5 nets/brand, 5 subsamples/net) that had been delivered for mass distribution in Papua New Guinea (PNG) having passed physiochemical testing of chemical content. Cone bioassays were performed on adjacent net pieces following WHO guidelines at the PNG Institute of Medical Research (PNGIMR) using pyrethroid susceptible Anopheles farauti s.s. and at Ifakara Health Institute (IHI), Tanzania using pyrethroid susceptible Anopheles gambiae s.s. Additionally, WHO tunnel tests was conducted at IHI on ITNs that did not meet cone bioefficacy thresholds. Results from IHI and PNGIMR were compared using Spearman's Rank, Bland Altman and Cohen's kappa. A literature review on the utility of cone bioassays for unused pyrethroid ITNs testing was also conducted.

\section{Results}

In cone bioassays, $13 / 20$ nets (65\%) met WHO bioefficacy criteria at IHI and 8/20 (40\%) at PNGIMR. All nets met WHO bioefficacy criteria on combined cone/tunnel tests. Results from IHI and PNGIMR correlated on 60-minute knockdown $\left(r_{s}=0.6, p=0.002, n=20\right)$ and 24 -hour mortality $\left(r_{s}=0.9, p<0.0001, n=20\right)$ but there was systematic bias between the results measured by Bland Altman. Of the 5 nets with discrepant result between IHI and PNGIMR, three had confidence intervals overlapping the $80 \%$ mortality threshold, with averages within $1-3 \%$ of the threshold. The agreement between the results to predict ITN failure was good with kappa=0.79 (0.53-1.00) and $90 \%$ accuracy.

\section{Conclusions}

WHO cone is a reproducible means to measure pyrethroid ITN bioefficacy using a combination of knockdown and mortality. In the absence of an alternative tests, cone tests could be used to assess the availability of active ingredients at the surface of ITN (where mosquitoes encounter it) as part of pre-delivery QA.

\section{Background}

Pyrethroid insecticide-treated nets (ITNs) are among the recommended public health interventions for control of malaria vectors [1] and are estimated to have prevented more than 450 million malaria cases between 2000 and 2015 [2]. While insecticide resistance [3] and mosquito behavioral changes [4] are factors contributing to the reduction of the effectiveness of pyrethroid ITNs, they can still provide a high degree of protection [5], especially in areas where anopheline mosquitoes are susceptible to pyrethroids like in Papua New Guinea (PNG) [3, 6].

It is important to deliver effective ITNs to protect those at risk against mosquito bites and malaria. To guarantee the effectiveness of ITNs distributed in malaria-endemic countries, it is necessary to conduct independent pre-delivery quality assurance (QA) and post-delivery operational monitoring of ITN quality [7]. ITN insecticide content, bioefficacy, physical integrity, and ITN survivorship are metrics used for ITN quality monitoring [8]. Bioefficacy is a measurement of the ability of the insecticide used on the ITN to induce mortality, knockdown (sublethal incapacitation) or prevent blood feeding of mosquitoes under laboratory conditions. Minimum bioefficacy thresholds for laboratory assays [8], have been set at a level that corresponded with malaria control, estimated by clinical trials conducted in Africa when mosquito vectors were still susceptible to pyrethroids [9]. Therefore, mosquito mortality benchmarks in place for ITNs are not only aimed to ensure personal protection to the user, but also that ITNs kill sufficient mosquitoes to provide community protection [10].

Pyrethroid ITN bioefficacy is evaluated experimentally under laboratory conditions with susceptible malaria vectors using cone bioassay and tunnel tests [8]. Bioefficacy evaluations provide reassurance of likely impact against susceptible vectors [8, 11-13]. New or unused pyrethroid ITNs should meet WHO standard bioefficacy criteria, i.e., $\geq 95 \%$ mosquitoes knockdown at 60-minutes (KD60) and/or $\geq 80 \%$ mortality at 24 hours (M24) for cone bioassays [8]. It has been shown by many studies that new or unused pyrethroid ITNs exhibit $100 \%$ for both or either of

Page $2 / 25$ 
these bioefficacy endpoint(s) [14-25]. The utility of cone bioassays is that they can estimate small variations in insecticide [17, 26] and bioefficacy [27] that can inform the effectiveness of the intervention. For pyrethroid ITNs unable to meet cone bioefficacy criteria, a second evaluation is conducted, using the WHO tunnel test that is designed for the evaluation of ITNs with blood feeding inhibition mode-of-action e.g. permethrin or etofenprox [9]. The performance thresholds for $\mathrm{WHO}$ tunnel tests are to induce $\geq 90 \%$ feeding inhibition (BFI) and/or $\geq 80 \%$ mortality at 24-hours (M24).

Physiochemical tests are currently used for ITN quality control [28] on the assumption that product performance is predictable based on the product specifications measured in predelivery inspections. Available evidence indicates that the vast majority of ITNs are likely to contain sufficient insecticide when they are delivered to households [29]. While this is encouraging, it should be remembered that predelivery inspections measure the total chemical content of the net yarn, while mosquitoes landing on the netting are exposed only to the insecticide present on the surface. The bioefficacy endpoints of KD60 or M24 are sensitive to small changes in insecticide surface concentration, with the critical ranges for bioefficacy differing between, and sometimes within, products [30]. Indeed, it has been shown that total insecticide content does not always correlate with bioefficacy [31].

Differences in ITN bioefficacy may be due to variations in spatial presentation and/or distribution of active ingredient within the netting, or the surface treatment as part of the manufacturing process. ITNs are manufactured mainly from polyester or polyethylene and careful product design, and manufacturing is required to ensure adequate bioavailability of active ingredient over the life of the product [30]. It is generally agreed that a validated, low-cost, easy-to-implement laboratory methodology -for assessing surface Al content is urgently needed for QA [29]. However, chemical assays of surface concentration such as the cyanopyrethroid field test and chemical tests such as high performance liquid chromatography and gas chromatography with mass spectrometry have not yet been found to correspond well to bioefficacy results [32].

To ensure successful malaria control, it is necessary to control bioefficacy of ITNs in pre-delivery inspections and/or prior to distribution. The cone bioassay remains the standard for determining pyrethroid ITN bioefficacy [8,33], as it is a simple and cost-effective methodology [20]. However, there is some debate about variability of cone bioassays results when different mosquito species are used. A robust test should ideally reveal reproducible bioefficacy results at different testing facilities on the same net samples against various Anopheles strains with

similar pyrethroid susceptibility levels. Not much is known about whether cone bioassay results are susceptible to systematic bias and random variability depending on the mosquito species used and other assay parameters not specified by the current WHO guidelines. This study explored utility of cone bioassay for pre-delivery QA in two test facilities using different mosquito species to test the assumption that cone bioassays are consistent and reproducible across locations, mosquito strains, and laboratories.

\section{Methods}

\section{Study design}

A double-blinded comparison of pyrethroid ITN bioefficacy as measured by WHO cone bioassay was conducted in two testing facilities. Twenty unused pyrethroid ITNs of 4 brands (100 subsamples, 5 subsamples per net) that had passed physiochemical inspection was assessed under laboratory conditions following WHO guidelines [8]. ITN subsamples were first evaluated using WHO cone bioassay and those that didn't meet the WHO cone assay performance criteria ( $\geq 95 \% \mathrm{KD} 60$ or $\geq 80 \%$ 24-hours mortality) were tested using the WHO tunnel test following standard procedures [8].

\section{Testing facilities}

The experiments were conducted at the Vector Control Product Testing Unit (VCPTU) of the Ifakara Health Institute (IHI) in Tanzania and at the Vector-borne Diseases Unit (VBDU) of the Papua New Guinea Institute of Medical Research (PNGIMR). The IHI facility is Good Laboratory Practice (GLP) accredited, SANAS G0033 [34].

Table 1 Summary of experiments conducted at Ifakara Health Institute (IHI) and Papua New Guinea Institute of Medical Research (PNGIMR) 


\begin{tabular}{lll} 
Experiment & Bioassay test in IHI & Cone bioassay test in PNGIMR \\
\hline Number of ITNs tested & 20 nets (100 net pieces) & 20 nets (100 net pieces) \\
\hline Mosquitoes exposed & 20 per net piece (cone bioassay) & 20 per net piece (cone bioassay) \\
\hline Experiment conditions & $27 \pm 1^{\circ} \mathrm{C}$ & $28 \pm 4^{\circ} \mathrm{C}$ \\
& $55 \%-82 \%$ pH & $53 \%-71 \% \mathrm{RH}$ \\
\hline Mosquito species & Pyrethroid susceptible ${ }^{*}$ An. gambiae s.s & Pyrethroid susceptible ${ }^{*}$ An. farautis.s \\
\hline Mosquito age & $3-5$ days (cone bioassay) & $2-5$ days (cone bioassay) \\
& $5-8$ days (tunnel tests) & \\
\hline WHO efficacy criteria & $\geq 95 \% \mathrm{KD} 60$ or $\geq 80 \% \mathrm{M} 24$ (cone bioassay) & $\geq 95 \% \mathrm{KD} 60$ or $\geq 80 \%$ M24 (cone bioassay) \\
\hline
\end{tabular}

*Sugar fed Anopheles gambiaes.s (Ifakara) and Anopheles farauti s.s were confirmed to be 100\% susceptible to alpha-cypermethrin, deltamethrin and permethrin insecticides at $1 \times$ WHO discriminating concentration at the time of evaluation. $\mathrm{RH}$ relative humidity; KD60 knockdown measured at 60 minutes (sublethal incapacitation); M24 mortality measured at 24 hours post exposure; FI feeding inhibition

\section{Description of tested products}

Five products (rectangular nets) were included in the study (Additional file 1).

i. PermaNet ${ }^{\circledR} 2.0$, a blue multi-filament polyester, 75 denier coated with $1.8 \mathrm{~g} / \mathrm{kg}\left(55 \mathrm{mg} / \mathrm{m}^{2}\right)$ deltamethrin and manufactured in 2019 by Vestergaard Frandsen, manufactured in Vietnam;

ii. PermaNet® 2.0, a yellow multi-filament polyester fiber, 75 denier coated with $1.8 \mathrm{~g} / \mathrm{kg}\left(55 \mathrm{mg} / \mathrm{m}^{2}\right)$ deltamethrin and manufactured in 2012 (manufacture location not given on label);

iii. Interceptor®, a blue multi-filament polyester fiber, 100 denier coated with $5 \mathrm{~g} / \mathrm{kg}\left(200 \mathrm{mg} / \mathrm{m}^{2}\right)$ alpha-cypermethrin and manufactured in 2020 by BASF in Thailand;

iv. SafeNet®, a blue multifilament polyester net, 100 denier coated with $5 \mathrm{~g} / \mathrm{kg}\left(200 \mathrm{mg} / \mathrm{m}^{2}\right)$ alpha-cypermethrin (manufactured in 2019 and 2020, location not given on label);

v. Yorkool ${ }^{\circledR}$, a blue multifilament polyester net, 100 denier coated with $1.8 \mathrm{~g} / \mathrm{kg}\left(55 \mathrm{mg} / \mathrm{m}^{2}\right)$ deltamethrin and manufactured in 2019 by Tianjin Yorkool International Trading Company limited, China.

Negative control net: untreated SafiNet ${ }^{\circledR}$ manufactured by $A$ to $Z$ textile mills, Tanzania and untreated Baomei® net manufactured in China were used in IHI and PNGIMR, respectively.

\section{Net origin and storage condition}

The PermaNet ${ }^{\circledR} 2.0$ manufactured in 2012 (PermaNet ${ }^{\circledR 2012)}$ nets were distributed in the year 2012 through the mass distribution campaign in PNG. These ITNs in unopened packaging were stored under tropical temperature and humidity in a store room of the Madang Provincial Health Authority between 2012 and 2018. The nets were transferred to a PNGIMR store in 2018 and kept at around $27^{\circ} \mathrm{C}$. Other ITNs i.e. the PermaNet ${ }^{\circledR} 2.0$ manufactured in 2019 (PermaNet ${ }^{\circledR} 2019$ ), the Interceptor ${ }^{\circledR}$, the SafeNet ${ }^{\circledR}$ and the Yorkool ${ }^{\circledR}$ were collected from shipping containers immediately upon arrival in PNG and prior to distribution, and stored a PNGIMR store room at around $27^{\circ} \mathrm{C}$.

\section{Net subsamples preparation and coding}

The sampled ITNs were labeled serially from 001 to 020 at PNGIMR. From these nets, ten net piece samples (25 cm x $25 \mathrm{~cm})$ were cut. Pairs of samples were cut from adjacent positions 1 to 5 as shown in (Figure 1) [8]. One net piece per position per net was sent to IHI and the second, adjacent piece was retained in PNG for testing. Thus, one hundred net pieces were each tested in PNGIMR and IHI in Tanzania. The five subsamples per net were given unique codes as A, B, C, D, and E, were wrapped individually in aluminum foil and stored in a temperaturecontrolled refrigerator at $4^{\circ} \mathrm{C}$. 
Subsamples were received in IHI in December 2020 from PNGIMR and immediately packed in new aluminum foil stored in a temperaturecontrolled refrigerator at $4^{\circ} \mathrm{C}$. The project investigators and facility technicians were blinded and unable to identify the products until the end of the study. After all experiments were completed and data were entered, data from PNGIMR cone bioassays was sent to IHI and the blinding was disclosed to the $\mathrm{IHI}$ investigators to match the results from the same type of study net types to enable analysis.

\section{Mosquito rearing and physiological status}

Tanzania: Nulliparous female pyrethroid susceptible An. gambiae s.s. (Ifakara strain) were used; sugar fed, aged between 3-5 days old in cone bioassays, and sugar starved for 6-8 hours, aged between 5-8 days old in WHO tunnel test. The mosquito colony is maintained according to MR4 Guidelines [35] at $27 \pm 2^{\circ} \mathrm{C}$ and relative humidity of $40 \%-100 \%$, with ambient (approximately 12:12) light dark cycle larvae are maintained on Tetramin fish flakes and adults are provided with $10 \%$ sucrose solution ad libitum and cow blood for egg laying.

PNG: Nulliparous female pyrethroid susceptible $A n$. farauti s.s. were used; sugar fed, aged between 2-5 days old in cone bioassays. The colony is maintained at $28 \pm 4^{\circ} \mathrm{C}$ and $68 \pm 25 \%$ relative humidity, with approximately $11 \mathrm{~h}$ dark and $12 \mathrm{~h} \mathrm{light}$ cycle, including a 30 min dusk and 30 min dawn period. The larvae are fed ground fish food (Marine Master Tropical Fish Flakes, Australia). The adults are provided $10 \%$ sucrose solution ad libitum and human blood for egg laying.

\section{Cone bioassay procedures}

On each net piece of $25 \mathrm{~cm}$ by $25 \mathrm{~cm}$, four standard WHO cones were fixed on a plastic cone board with holes cut and held at $60^{\circ}[36]$ in $\mathrm{IHI}$, Tanzania (Figure 2A) to maximize space and mosquito contact with the ITN and on a board at $45^{\circ}$ [8] in PNGIMR (Figure 2B) according to WHO guidelines. Net pieces were taken from the fridge and kept at room temperature for 2 hours before testing. Five laboratory-reared susceptible mosquitoes were exposed into each cone for 3-minutes after which, mosquitoes were removed gently from the cones using a mouth aspirator and kept in individually labeled paper cups, one for each cone. Mosquitoes were provided with cotton wool moistened with $10 \%$ sucrose solution. Four replicates of 5 mosquitoes were performed on each of the five net pieces making a total of 100 mosquitoes exposed per net. Mosquito knockdown was recorded after 60-minutes (KD60) and mortality after 24-hours (M24). An untreated net was used as negative control. The bioassays and holding period were carried out at $27 \pm 1^{\circ} \mathrm{C}$ and at $55 \%-82 \%$ relative humidity in Tanzania and $28 \pm 4{ }^{\circ} \mathrm{C}$ and at $53 \%-71 \%$ relative humidity in PNG. If the M24 exceeded $10 \%$ in a negative control, the test was repeated and if the mortality in a negative control was equal or below $10 \%$, the results were adjusted using Abbott's formula [8].

\section{Tunnel test procedures}

WHO tunnel tests were only performed in IHI Tanzania because tunnel tests are not currently established at PNGIMR. Two out of five subsamples of a particular net that did not meet the WHO cone efficacy criteria, were selected for the WHO tunnel test against susceptible Anopheles gambiae s.s. These were subsamples that gave mortality closest to the average mortality in the cone test of five subsamples. Tunnel tests were conducted following WHO guidelines [8]. Non-blood fed nulliparous females 5-8 days old, sugar starved for 6-8 hours were released in a tunnel made of glass, $60 \mathrm{~cm}$ length. At each end of the tunnel, a $25-\mathrm{cm}$ square mosquito cage covered with polyester netting was fitted. At one third of the length, a $25 \mathrm{~cm} \times 25 \mathrm{~cm}$ swatch of netting sample was affixed. The surface of netting "available" to mosquitoes is $400 \mathrm{~cm}^{2}(20 \mathrm{~cm} \times 20 \mathrm{~cm})$, with $9 \times 1 \mathrm{~cm}$ in diameter holes: one hole is located at the centre of the square; the other eight are equidistant and located at $5 \mathrm{~cm}$ from the border. In the shorter section of the tunnel, a small rabbit, shaved on its back and restrained in a mesh tunnel was placed as bait. In the cage at the end of the longer section of the tunnel, 100 female mosquitoes were introduced at 21:00 hours. The following morning at 09:00 hours, the mosquitoes were removed using a mouth aspirator and counted separately from each section of the tunnel, and mortality and blood feeding rates (feeding inhibition FI) were recorded. The mosquitoes were placed in paper cups and provided with cotton wool moistened with 10\% sugar solution. Mortality 24 hours (M24) was recorded at around 09:00 hours the following day. Mosquitoes exposed to untreated nets were used as controls to monitor the quality of the bioassay. The bioassays and holding period were carried out at $27^{\circ} \mathrm{C} \pm 2{ }^{\circ} \mathrm{C}$ and $60 \%-100 \%$ relative humidity. Overall mortality was measured by pooling the mortalities of mosquitoes from the two sections of the tunnel. Acceptable feeding success and M24 in controls were $50 \%$ and $10 \%$, respectively. Any tests below the specified control cut off were repeated.

\section{Data management and statistical analyses}

Paper data collection sheets were used to record data, which was double-entered in Microsoft Excel®. All data was analyzed using Stata ${ }^{\circledR}$ statistical package version 14 (Stata Statistical Software: Release 14. College Station, TX: StataCorp LP. StataCorp. 2013). Proportional KD60

Page 5/25 
and M24 or Fl and M24 were presented as arithmetic means with their respective $95 \%$ confidence intervals (Cl). Pass or fail for each net was calculated based on WHO standard efficacy criteria i.e. $\geq 95 \%$ KD60 and/or $\geq 80 \% \mathrm{M} 24$ for cone assay; $\geq 90 \% \mathrm{FI}$ and/or $\geq 80 \% \mathrm{M} 24$ for WHO tunnel test. Linear regression was used to analyze the relationship between KD60 and M24. The Spearman rank correlation coefficient ( $r_{s}$ ) was calculated to estimate the level of correlation between IHI and PNGIMR on cone bioassay results (KD60 and M24). Bland-Altman methods [37] were used to assess the agreement between of KD60 and M24 cone assay results from IHI and PNGIMR testing facilities, respectively. The Cohen's kappa (k) was used to assess the agreement of cone assay tests on the evaluated nets against standard efficacy criteria (pass/fail rate).

\section{Literature review on utility of cone bioassay for unused pyrethroid ITNs testing}

A search of the literature on ITN efficacy studies, durability studies or WHOPES (World Health Organisation Pesticide Evaluation Scheme) specification reports published between 2001 and 2021 was conducted in October, 2021 in PubMed and Global Health using the keywords "bio-efficacy" or "cone bioassay tests" and "tunnel tests" or "Insecticide treated nets" and "long lasting insecticidal nets" and Google Scholar using the keyword "WHOPES working group meeting". The 2,362 titles were identified; PubMed 87 titles, Global Health 1,604 titles and Google Scholar 671 titles and of these, seventy publications were fully screened and sixty were included in the review. The criteria for selection were reports using standard WHO evaluation methods on unused pyrethroid ITNs with Anopheles mosquitoes that reported both KD60 and M24.

\section{Results}

\section{Bioefficacy of unused ITNs against susceptible An. gambiae in IHI Tanzania}

All 20 nets met the WHO bioefficacy pass criteria based on the combined cone and tunnel tests, Table 2 . In the cone bioassay, $13 / 20$ nets (65\%) met WHO cone bioefficacy criteria of $\geq 95 \% \mathrm{KD} 60$ and/or $\geq 80 \% \mathrm{M} 24$. The seven nets that did not meet cone bioassay criteria, met bioefficacy criteria of $\geq 90 \% \mathrm{FI}$ and $\geq 80 \% \mathrm{M} 24$ in the WHO tunnel tests.

Bioefficacy of the unused ITNs against susceptible An. farauti in PNGIMR

In the cone bioassay, $8 / 20$ nets (40\%) met WHO cone bioefficacy criteria of $\geq 95 \%$ KD 60 and/or $\geq 80 \%$ M24, Table 2 .

\section{The relationship between 60-minute knockdown and 24-hours mortality in cone bioassay}

In $\mathrm{IHI}$, the relationship between knockdown and mortality measured by regression was 0.36 (95\% $\mathrm{Cl}: 0.15-0.57 p=0.002)$ indicating that $\mathrm{KD} 60$ and M24 were not closely related. While in PNGIMR the relationship was 0.78 (95\% Cl: $0.64-0.93 p<0.001)$ indicating that there was high probability of knocked down mosquitoes dying. 
Table 2

WHO cone bioassay results on tested unused pyrethroid ITNs in IHI Tanzania (with susceptible Anopheles gambiae s.s.) and PNGIMR (with susceptible Anopheles farauti s.s.) testing facilities

\begin{tabular}{|c|c|c|c|c|c|c|}
\hline \multirow[b]{2}{*}{ Test Item } & \multicolumn{3}{|l|}{ Cone test } & \multicolumn{2}{|l|}{ Tunnel test* } & \multirow{2}{*}{$\begin{array}{l}\text { \#Nets pass combined cone and } \\
\text { tunnel tests }\end{array}$} \\
\hline & $\begin{array}{l}\% \text { KD60 } \\
(95 \% \mathrm{Cl})\end{array}$ & $\begin{array}{l}\text { \%24-hr } \\
\text { Mortality } \\
(95 \% \mathrm{Cl})\end{array}$ & $\begin{array}{l}\text { \#Nets pass } \\
\text { in cone }\end{array}$ & $\begin{array}{l}\% \text { Feeding inhibition } \\
(95 \% \mathrm{Cl})\end{array}$ & $\begin{array}{l}\text { \%24-hr } \\
\text { Mortality(95\% Cl) }\end{array}$ & \\
\hline \multicolumn{7}{|l|}{$\mathrm{IH}$} \\
\hline PermaNet ${ }^{\circledR}+$ & 100 & $\begin{array}{l}99.7(99.2- \\
100)\end{array}$ & $4 / 4$ & & & $4 / 4$ \\
\hline PermaNet ${ }^{\circledR} \#$ & $\begin{array}{l}80.0(76.0- \\
84.0)\end{array}$ & $\begin{array}{l}22.3(17.8- \\
26.7)\end{array}$ & $1 / 4$ & $98.3(94.7-100)$ & $97.8(94.5-100)$ & $4 / 4$ \\
\hline Interceptor® & $\begin{array}{l}85.8 \text { (82.6- } \\
88.9)\end{array}$ & $\begin{array}{l}37.9(32.0- \\
43.7)\end{array}$ & $1 / 4$ & $99.7(99-100)$ & $99.5(98.5-100)$ & $4 / 4$ \\
\hline SafeNet ${ }^{\circledR}$ & $\begin{array}{l}97.3(95.6- \\
98.9)\end{array}$ & $\begin{array}{l}61.1(55.2- \\
67.0)\end{array}$ & $3 / 4$ & 100 & 100 & $4 / 4$ \\
\hline Yorkool® & $\begin{array}{l}96.8(94.8- \\
98.7)\end{array}$ & $\begin{array}{l}59.7(56.1- \\
63.3)\end{array}$ & $4 / 4$ & & & $4 / 4$ \\
\hline \multicolumn{7}{|l|}{ PNGIMR } \\
\hline PermaNet ${ }^{\circledR}+$ & $\begin{array}{l}96.4(92.3- \\
100)\end{array}$ & $\begin{array}{l}99.6(98.8- \\
100)\end{array}$ & $4 / 4$ & & & \\
\hline PermaNet ${ }^{\circledR} \#$ & $\begin{array}{l}37.1(29.3- \\
44.9)\end{array}$ & $\begin{array}{l}25.9(14.1- \\
37.6)\end{array}$ & $0 / 4$ & & & \\
\hline Interceptor ${ }^{\circledR}$ & $\begin{array}{l}79.3(72.7- \\
85.8)\end{array}$ & $\begin{array}{l}72.8(66.7- \\
78.8)\end{array}$ & $0 / 4$ & & & \\
\hline SafeNet ${ }^{\circledR}$ & $\begin{array}{l}82.0(75.1- \\
88.9)\end{array}$ & $\begin{array}{l}81.0(74.8- \\
87.2)\end{array}$ & $1 / 4$ & & & \\
\hline Yorkool® & $\begin{array}{l}87.3(83.5- \\
91.0)\end{array}$ & $\begin{array}{l}88.5(83.9- \\
93.1)\end{array}$ & $3 / 4$ & & & \\
\hline
\end{tabular}

* Tunnel test performed to the nets that did not meet optimal efficacy criteria ( $\geq 95 \% \mathrm{KD} 60$ and/or $\geq 80 \% \mathrm{M} 24$ ) in cone bioassay at IHI,

+ PermaNet® 2.0 manufactured in 2012,

\# PermaNet® 2.0 manufactured in 2019.

Level of correlation between IHI and PNGIMR on cone bioassay results (KD60 and M24)

There was a correlation between IHI and PNGIMR results with a stronger association between $\mathrm{M} 24$ results $(\mathrm{r}=0.9, p<0.0001, \mathrm{n}=20)$ than $\mathrm{KD} 60$ $(\mathrm{r}=0.6, p=0.002, \mathrm{n}=20)$ (Figure 3).

\section{Agreement of cone bioassay at IHI and PNGIMR testing facilities based on KD60 and M24}

Using Bland-Altman more consistent agreement in M24 results than for KD60 (Figure 4). The limits of agreement for both endpoints were wide: KD60 mean difference (limits of agreement) 15.5 (-25.4 to 56.5) and M24-17.0 (-61.4 to 27.3). As expected, agreement was highest among the most efficacious nets with high KD60 and M24.

\section{Agreement of cone bioassay at IHI and PNGIMR testing facilities based on WHO pass/fail criteria}

Nets that exceeded KD60 or M24 thresholds or had mean values close to the threshold with confidence intervals exceeding the threshold were categorized as pass (Figure 5). The IHI and PNGIMR data agreed for $n=18$ (90\%) of the test samples, classifying $n=6$ (30\%) as failed at both facilities and $n=12(60 \%)$ as pass at both facilities, Table 3 . The agreement between the results at IHI and PNGMR based on WHO bioefficacy criteria (pass/fail rate) to predict ITN failure was good with $\mathrm{k}=0.79(0.53-1.00)$ and $90 \%$ accuracy. The two discrepant nets (net 5 and net 12 ) failed at PNGIMR but passed at IHI on KD60 (Figure 5). 
No nets with M24 exceeding $80 \%$ failed at either facility, while the majority of nets that passed at IHI, passed only on KD60 (Figure 6 ). It should be noted that the $80 \% \mathrm{M} 24$ and 95\% KD60 thresholds themselves are subject to stochastic variation. If tests are done using 100 mosquitoes per net as per WHO guidelines, we expect an assay-inherent $95 \% \mathrm{Cl}$ of $71 \%$ and $87 \%$ around the $80 \%$ mortality threshold and a $95 \% \mathrm{Cl}$ of $89 \%$ and $98 \%$ around the $95 \%$ KD 60 threshold (Figure 6).

Table 3

Contingency analysis for cone bioassays conducted in $\mathrm{IHI}$ and PNGIMR to classify the $n=20$ ITNs into 'pass' and 'fail' categories based on cone bioassay result.

\begin{tabular}{|lllll|}
\hline \multicolumn{5}{|c|}{ PNGIMR } \\
\hline & Pass N (\%) & Fail N (\%) & Total N (\%) \\
\hline IHI & Pass & $12^{\mathrm{a}}(100)$ & $2^{\mathrm{c}}(25)$ & $14(70)$ \\
\cline { 2 - 4 } & Fail & $0^{\mathrm{b}}(0)$ & $6^{\mathrm{d}}(75)$ & $6(30)$ \\
\hline & Total & $12(60)$ & $8(40)$ & $20(100)$ \\
\hline
\end{tabular}

'a' and 'd' the number of nets agreed results at both testing facilities, ' $b$ ' and 'c' the number of nets with discrepant results between testing facilities

\section{Literature review on utility of cone bioassay for pyrethroid ITNs testing}

The literature review on utility of cone bioassay for pyrethroid ITN testing showed most unwashed nets score high KD60 and M24 (Figure 7). Publications with lower efficacy ITNs were observed from some country monitoring reports, in WHOPES supervised studies and limited number of published articles. From a regression conducted on 83 observations included both KD60 and M24 mainly with An. gambiae s.s (63/83) and ITNs mainly deltamethrin impregnated (51/83) showed excellent knockdown and mortality in unwashed nets with only 12 published examples of new ITNs with knockdown below 95\%, Table 4 In the published examples of new nets average knockdown was 96\% (95\% Cl: 94-98) and mortality was $92 \%$ (95\% Cl: 88-96). Interestingly, even permethrin ITNs gave very high knockdown 89\% (95\% Cl: 74-100) and mortality 89\% (95\% Cl: 68-100) in studies published between 2008 and 2017. 
Table 4

The literature review on cone bioassays for pyrethroid ITN testing

\begin{tabular}{|c|c|c|c|c|c|c|c|c|}
\hline Author/Report & Location & $\begin{array}{l}\text { Pyrethroid } \\
\text { ITN }^{\star}\end{array}$ & $\begin{array}{l}\text { Active } \\
\text { Ingredients }\end{array}$ & $\begin{array}{l}\text { Production } \\
\text { technology }\end{array}$ & Year" & $\begin{array}{l}\text { Mosquito strains } \\
\text { (Susceptible) }\end{array}$ & KD60 & M24 \\
\hline $\begin{array}{l}\text { Abilio and } \\
\text { colleagues, } 2015 \\
\text { [21] }\end{array}$ & Mozambique & Interceptor® & $\begin{array}{l}\text { Alpha- } \\
\text { cypermethrin }\end{array}$ & Impregnation & & An. arabiensis & 80.56 & 98.84 \\
\hline $\begin{array}{l}\text { Abilio and } \\
\text { colleagues, } 2015 \\
\text { [21] }\end{array}$ & Mozambique & $\begin{array}{l}\text { PermaNet® } \\
2.0\end{array}$ & Deltamethrin & Impregnation & & An. arabiensis & 94.72 & 100 \\
\hline $\begin{array}{l}\text { Abilio and } \\
\text { colleagues, } 2015 \\
\text { [21] }\end{array}$ & Mozambique & Olyset Net巴 & Permethrin & Incorporation & & An. arabiensis & 68.33 & 90.36 \\
\hline $\begin{array}{l}\text { Agossa and } \\
\text { colleagues, } 2014 \\
\text { [62] }\end{array}$ & Benin & $\begin{array}{l}\text { PermaNet }{ }^{\circledR} \\
2.0\end{array}$ & Deltamethrin & Impregnation & & $\begin{array}{l}\text { An. gambiae } \\
\text { (Kisumu strain) }\end{array}$ & 100 & 100 \\
\hline $\begin{array}{l}\text { Ahogni and } \\
\text { colleagues, } 2019 \\
\text { [38] }\end{array}$ & Benin & Yorkool® & Deltamethrin & Impregnation & 2017 & $\begin{array}{l}\text { An. gambiae } \\
\text { (Kisumu strain) }\end{array}$ & 62 & 74 \\
\hline $\begin{array}{l}\text { Allossogbe and } \\
\text { colleagues, } 2017 \\
\text { [63] }\end{array}$ & Benin & $\begin{array}{l}\text { PermaNet }{ }^{\circledR} \\
2.0\end{array}$ & Deltamethrin & Impregnation & $\begin{array}{l}2015- \\
2016\end{array}$ & $\begin{array}{l}\text { An. gambiae } \\
\text { (Kisumu strain) }\end{array}$ & 93.33 & 100 \\
\hline $\begin{array}{l}\text { Allossogbe and } \\
\text { colleagues, } 2017 \\
\text { [63] }\end{array}$ & Benin & Olyset Net ${ }^{\circledR}$ & Permethrin & Incorporation & $\begin{array}{l}2015- \\
2016\end{array}$ & $\begin{array}{l}\text { An. gambiae } \\
\text { (Kisumu strain) }\end{array}$ & 100 & 100 \\
\hline $\begin{array}{l}\text { Bagheri and } \\
\text { colleagues, } 2017 \\
\text { [39] }\end{array}$ & Iran & $\begin{array}{l}\text { PermaNet® } \\
2.0\end{array}$ & Deltamethrin & Impregnation & 2016 & An. stephensi & 74 & 22 \\
\hline $\begin{array}{l}\text { Bhatt and } \\
\text { colleagues, } 2012 \\
\text { [23] }\end{array}$ & India & Interceptor® & $\begin{array}{l}\text { Alpha- } \\
\text { cypermethrin }\end{array}$ & Impregnation & 2006 & An. culicifacies & 96.7 & 100 \\
\hline $\begin{array}{l}\text { Camara and } \\
\text { colleagues, } 2018 \\
\text { [64] }\end{array}$ & Côte d'Ivoire & Interceptor ${ }^{\circledR}$ & $\begin{array}{l}\text { Alpha- } \\
\text { cypermethrin }\end{array}$ & Impregnation & & $\begin{array}{l}\text { An. gambiae } \\
\text { (Kisumu strain) }\end{array}$ & 97 & 99 \\
\hline $\begin{array}{l}\text { Castellanos and } \\
\text { colleagues, } 2021 \\
{[18]}\end{array}$ & Guatemala & $\begin{array}{l}\text { PermaNet® } \\
2.0\end{array}$ & Deltamethrin & Impregnation & 2012 & An. albimanus & 100 & 100 \\
\hline $\begin{array}{l}\text { Clegban and } \\
\text { colleagues, } 2021 \\
\text { [65] }\end{array}$ & Côte d'Ivoire & Yahe ${ }^{\circledR}$ & Deltamethrin & Impregnation & 2014 & $\begin{array}{l}\text { An. gambiae } \\
\text { (Kisumu strain) }\end{array}$ & 100 & 97.2 \\
\hline $\begin{array}{l}\text { Clegban and } \\
\text { colleagues, } 2021 \\
\text { [65] }\end{array}$ & Côte d'Ivoire & $\begin{array}{l}\text { PandaNet } \AA \\
2.0\end{array}$ & Deltamethrin & Incorporation & 2014 & $\begin{array}{l}\text { An. gambiae } \\
\text { (Kisumu strain) }\end{array}$ & 100 & 100 \\
\hline $\begin{array}{l}\text { Graham and } \\
\text { colleagues, } 2005 \\
\text { [17] }\end{array}$ & Iran & $\begin{array}{l}\text { PermaNet® } \\
2.0\end{array}$ & Deltamethrin & Impregnation & 2000 & $\begin{array}{l}\text { An. stephensi } \\
\text { (Beech strain) }\end{array}$ & 100 & 97.7 \\
\hline $\begin{array}{l}\text { Kilian and } \\
\text { colleagues, } 2008 \\
\text { [43] }\end{array}$ & $\begin{array}{l}\text { Montpellier, } \\
\text { France }\end{array}$ & $\begin{array}{l}\text { PermaNet } \AA \\
1.0\end{array}$ & Deltamethrin & Impregnation & 2000 & $\begin{array}{l}\text { An. gambiae } \\
\text { (Kisumu strain) }\end{array}$ & 95 & 80 \\
\hline $\begin{array}{l}\text { Kilian and } \\
\text { colleagues, } 2008 \\
\text { [43] }\end{array}$ & $\begin{array}{l}\text { CDC Atlanta, } \\
\text { USA }\end{array}$ & $\begin{array}{l}\text { PermaNet }{ }^{\circledR} \\
2.0\end{array}$ & Deltamethrin & Impregnation & 2002 & $\begin{array}{l}\text { An. gambiae } \\
\text { (Kisumu strain) }\end{array}$ & 95 & 80 \\
\hline $\begin{array}{l}\text { Kweka and } \\
\text { colleagues, } 2011 \\
\text { [66] }\end{array}$ & Tanzania & $\begin{array}{l}\text { PermaNet }{ }^{\circledR} \\
2.0\end{array}$ & Deltamethrin & Impregnation & 2005 & $\begin{array}{l}\text { An. gambiae } \\
\text { (Kisumu strain) }\end{array}$ & 100 & 100 \\
\hline
\end{tabular}

* Include new unused, old unused or unwashed Long-Lasting Insecticidal Nets; \#Many reviewed studies did not report ITNs manufactured date/year, hence in this review encompass and report either manufactured year/distributed year/tested year or date of meeting; KD60 knockdown measured at 60 minutes (sublethal incapacitation); M24 mortality measured at 24 hours post exposure. 


\begin{tabular}{|c|c|c|c|c|c|c|c|c|}
\hline Author/Report & Location & $\begin{array}{l}\text { Pyrethroid } \\
\text { ITN }^{*}\end{array}$ & $\begin{array}{l}\text { Active } \\
\text { Ingredients }\end{array}$ & $\begin{array}{l}\text { Production } \\
\text { technology }\end{array}$ & Year" & $\begin{array}{l}\text { Mosquito strains } \\
\text { (Susceptible) }\end{array}$ & KD60 & M24 \\
\hline $\begin{array}{l}\text { Kweka and } \\
\text { colleagues, } 2017 \\
\text { [67] }\end{array}$ & Tanzania & $\begin{array}{l}\text { PermaNet }{ }^{\circledR} \\
2,0\end{array}$ & Deltamethrin & Impregnation & & $\begin{array}{l}\text { An. gambiae } \\
\text { (Kisumu strain) }\end{array}$ & 100 & 100 \\
\hline $\begin{array}{l}\text { Kweka and } \\
\text { colleagues, } 2019 \\
\text { [68] }\end{array}$ & Tanzania & MagNet巴 & $\begin{array}{l}\text { Alpha- } \\
\text { cypermethrin }\end{array}$ & Incorporation & & $\begin{array}{l}\text { An. gambiae } \\
\text { (Kisumu strain) }\end{array}$ & 90.4 & 100 \\
\hline $\begin{array}{l}\text { Kweka and } \\
\text { colleagues, } 2019 \\
\text { [68] }\end{array}$ & Tanzania & DuraNet ${ }^{\circledR}$ & $\begin{array}{l}\text { Alpha- } \\
\text { cypermethrin }\end{array}$ & Incorporation & & $\begin{array}{l}\text { An. gambiae } \\
\text { (Kisumu strain) }\end{array}$ & 100 & 100 \\
\hline $\begin{array}{l}\text { Mahande and } \\
\text { colleagues, } 2018 \\
\text { [69] }\end{array}$ & Tanzania & DuraNet ${ }^{\circledR}$ & $\begin{array}{l}\text { Alpha- } \\
\text { cypermethrin }\end{array}$ & Incorporation & 2015 & $\begin{array}{l}\text { An. gambiae } \\
\text { (Kisumu strain) }\end{array}$ & 100 & 100 \\
\hline $\begin{array}{l}\text { Malima and } \\
\text { colleagues, } 2013 \\
\text { [22] }\end{array}$ & Tanzania & Interceptor® & $\begin{array}{l}\text { Alpha- } \\
\text { cypermethrin }\end{array}$ & Impregnation & & An. gambiae s.I & 100 & 100 \\
\hline $\begin{array}{l}\text { Mussa and } \\
\text { colleagues, } 2020 \\
\text { [70] }\end{array}$ & Tanzania & DawaPlus ${ }^{\circledR}$ & Deltamethrin & Impregnation & 2019 & An. gambiae & 100 & 92.5 \\
\hline $\begin{array}{l}\text { Ngufor and } \\
\text { colleagues, } 2020 \\
\text { [71] }\end{array}$ & Benin & $\begin{array}{l}\text { Royal } \\
\text { Sentry }\end{array}$ & $\begin{array}{l}\text { Alpha- } \\
\text { cypermethrin }\end{array}$ & Incorporation & & $\begin{array}{l}\text { An. gambiae } \\
\text { (Kisumu strain) }\end{array}$ & 100 & 98 \\
\hline $\begin{array}{l}\text { Okia and } \\
\text { colleagues, } 2013 \\
\text { [16] }\end{array}$ & Uganda & $\begin{array}{l}\text { PermaNet }{ }^{\circledR} \\
2.0\end{array}$ & Deltamethrin & Impregnation & $\begin{array}{l}\text { Started } \\
2011\end{array}$ & $\begin{array}{l}\text { An. gambiae } \\
\text { (Kisumu strain) }\end{array}$ & 100 & 100 \\
\hline $\begin{array}{l}\text { Okia and } \\
\text { colleagues, } 2013 \\
\text { [16] }\end{array}$ & Uganda & Interceptor® & $\begin{array}{l}\text { Alpha- } \\
\text { cypermethrin }\end{array}$ & Impregnation & $\begin{array}{l}\text { Started } \\
2011\end{array}$ & $\begin{array}{l}\text { An. gambiae } \\
\text { (Kisumu strain) }\end{array}$ & 95 & 100 \\
\hline $\begin{array}{l}\text { Okia and } \\
\text { colleagues, } 2013 \\
{[16]}\end{array}$ & Uganda & Olyset $\mathrm{Net}{ }^{\circledR}$ & Permethrin & Incorporation & $\begin{array}{l}\text { started } \\
2011\end{array}$ & $\begin{array}{l}\text { An. gambiae } \\
\text { (Kisumu strain) }\end{array}$ & 100 & 100 \\
\hline $\begin{array}{l}\text { Pennetier and } \\
\text { colleagues, } 2013 \\
\text { [72] }\end{array}$ & $\begin{array}{l}\text { Malanville, } \\
\text { Benin }\end{array}$ & Olyset $\mathrm{Net}{ }^{\circledR}$ & Permethrin & Incorporation & & $\begin{array}{l}\text { An. gambiae } \\
\text { (Kisumu strain) }\end{array}$ & 64 & 100 \\
\hline $\begin{array}{l}\text { Rafinejad and } \\
\text { colleagues, } 2008 \\
\text { [20] }\end{array}$ & Iran & PermaNet ${ }^{\circledR}$ & Deltamethrin & Impregnation & & An. stephensi & 100 & 94.9 \\
\hline $\begin{array}{l}\text { Rafinejad and } \\
\text { colleagues, } 2008 \\
\text { [20] }\end{array}$ & Iran & Olyset $\mathrm{Net}{ }^{\circledR}$ & Permethrin & Incorporation & & An. stephensi & 100 & 97 \\
\hline $\begin{array}{l}\text { Randriamaherijaona } \\
\text { and colleagues, } \\
2017 \text { [40] }\end{array}$ & Madagascar & $\begin{array}{l}\text { Royal } \\
\text { Sentry® }\end{array}$ & $\begin{array}{l}\text { Alpha- } \\
\text { cypermethrin }\end{array}$ & Incorporation & & An. arabiensis & 100 & 90.2 \\
\hline $\begin{array}{l}\text { Sood and } \\
\text { colleagues, } 2011 \\
\text { [73] }\end{array}$ & India & $\begin{array}{l}\text { PermaNet } \circledast \\
2.0\end{array}$ & Deltamethrin & Impregnation & & An. stephensi & 100 & 100 \\
\hline $\begin{array}{l}\text { Sood and } \\
\text { colleagues, } 2011 \\
\text { [73] }\end{array}$ & India & Olyset $\mathrm{Net}{ }^{\circledR}$ & Permethrin & Incorporation & & An. stephensi & 100 & 100 \\
\hline $\begin{array}{l}\text { Vinit and } \\
\text { colleagues, } 2020 \\
\text { [41] }\end{array}$ & $\begin{array}{l}\text { Papua New } \\
\text { Guinea }\end{array}$ & $\begin{array}{l}\text { PermaNet } \AA \\
2.0\end{array}$ & Deltamethrin & Impregnation & $\begin{array}{l}\text { Between } \\
2007 \text { and } \\
2012\end{array}$ & An. farauti & 96.48 & 98.72 \\
\hline $\begin{array}{l}\text { Vinit and } \\
\text { colleagues, } 2020 \\
\text { [41] }\end{array}$ & $\begin{array}{l}\text { Papua New } \\
\text { Guinea }\end{array}$ & $\begin{array}{l}\text { PermaNet }{ }^{\circledR} \\
2.0\end{array}$ & Deltamethrin & Impregnation & $\begin{array}{l}\text { Between } \\
2013 \text { and } \\
2019\end{array}$ & An. farauti & 41.23 & 40.12 \\
\hline
\end{tabular}

* Include new unused, old unused or unwashed Long-Lasting Insecticidal Nets; ${ }^{\#}$ Many reviewed studies did not report ITNs manufactured date/year, hence in this review encompass and report either manufactured year/distributed year/tested year or date of meeting; KD60 knockdown measured at 60 minutes (sublethal incapacitation); M24 mortality measured at 24 hours post exposure. 


\begin{tabular}{|c|c|c|c|c|c|c|c|c|}
\hline Author/Report & Location & $\begin{array}{l}\text { Pyrethroid } \\
\text { ITN }^{*}\end{array}$ & $\begin{array}{l}\text { Active } \\
\text { Ingredients }\end{array}$ & $\begin{array}{l}\text { Production } \\
\text { technology }\end{array}$ & Year"\# & $\begin{array}{l}\text { Mosquito strains } \\
\text { (Susceptible) }\end{array}$ & KD60 & M24 \\
\hline WHO, 2004 [74] & $\begin{array}{l}\text { Montipellier, } \\
\text { France }\end{array}$ & $\begin{array}{l}\text { PermaNet }{ }^{\circledR} \\
1.0\end{array}$ & Deltamethrin & Impregnation & $\begin{array}{l}2-4 \\
\text { December, } \\
2003\end{array}$ & $\begin{array}{l}\text { An. gambiae } \\
\text { (Kisumu strain) }\end{array}$ & 100 & 100 \\
\hline WHO, 2004 [74] & Benin & $\begin{array}{l}\text { PermaNet }{ }^{\circledR} \\
2.0\end{array}$ & Deltamethrin & Impregnation & $\begin{array}{l}2-4 \\
\text { December, } \\
2003\end{array}$ & $\begin{array}{l}\text { An. gambiae } \\
\text { (Kisumu strain) }\end{array}$ & 100 & 100 \\
\hline WHO, 2004 [74] & $\begin{array}{l}\text { Montipellier, } \\
\text { France }\end{array}$ & $\begin{array}{l}\text { PermaNet }{ }^{\circledR} \\
1.0\end{array}$ & Deltamethrin & Impregnation & $\begin{array}{l}2-4 \\
\text { December, } \\
2003\end{array}$ & $\begin{array}{l}\text { Cx. } \\
\text { quinquefasciatus }\end{array}$ & 100 & 100 \\
\hline WHO, 2007 [33] & $\begin{array}{l}\text { Malanville, } \\
\text { Benin }\end{array}$ & Interceptor ${ }^{\circledR}$ & $\begin{array}{l}\text { Alpha- } \\
\text { cypermethrin }\end{array}$ & Impregnation & $\begin{array}{l}11-14 \\
\text { December, } \\
2006\end{array}$ & An. gambiae & 100 & 100 \\
\hline WHO, 2007 [33] & $\begin{array}{l}\text { Montipellier, } \\
\text { France }\end{array}$ & $\begin{array}{l}\text { Hiking } \\
\text { Group® }\end{array}$ & Deltamethrin & Impregnation & $\begin{array}{l}11-14 \\
\text { December, } \\
2006\end{array}$ & $\begin{array}{l}\text { An. gambiae } \\
\text { (Kisumu) }\end{array}$ & 100 & 95 \\
\hline WHO, 2007 [33] & $\begin{array}{l}\text { Montipellier, } \\
\text { France }\end{array}$ & Yorkool® & Deltamethrin & Impregnation & $\begin{array}{l}11-14 \\
\text { December, } \\
2006\end{array}$ & $\begin{array}{l}\text { An. gambiae } \\
\text { (Kisumu) }\end{array}$ & 83 & 16 \\
\hline WHO, 2007 [33] & $\begin{array}{l}\text { Montipellier, } \\
\text { France }\end{array}$ & $\begin{array}{l}\text { Netto } \\
\text { Group }{ }^{\circledR}\end{array}$ & Deltamethrin & Impregnation & $\begin{array}{l}11-14 \\
\text { December, } \\
2006\end{array}$ & $\begin{array}{l}\text { An. gambiae } \\
\text { (Kisumu) }\end{array}$ & 95 & 100 \\
\hline WHO, 2007 [33] & $\begin{array}{l}\text { Montipellier, } \\
\text { France }\end{array}$ & $\begin{array}{l}\text { PermaNet } \AA \\
2.0\end{array}$ & Deltamethrin & Impregnation & $\begin{array}{l}11-14 \\
\text { December, } \\
2006\end{array}$ & $\begin{array}{l}\text { An. gambiae } \\
\text { (Kisumu) }\end{array}$ & 100 & 100 \\
\hline WHO, 2008 [12] & $\begin{array}{l}\text { Kyenjonjo, } \\
\text { Uganda }\end{array}$ & $\begin{array}{l}\text { PermaNet }{ }^{\circledR} \\
2.0\end{array}$ & Deltamethrin & Impregnation & $\begin{array}{l}11-14 \\
\text { December, } \\
2006\end{array}$ & $\begin{array}{l}\text { An. gambiae } \\
\text { (Kisumu) }\end{array}$ & 95 & 95 \\
\hline WHO, 2008 [12] & $\begin{array}{l}\text { Montipellier, } \\
\text { France }\end{array}$ & Dawaplus ${ }^{\circledR}$ & Deltamethrin & Impregnation & $\begin{array}{l}10-13 \\
\text { December, } \\
2007\end{array}$ & $\begin{array}{l}\text { An. gambiae } \\
\text { (Kisumu) }\end{array}$ & 93 & 39 \\
\hline WHO, 2008 [12] & $\begin{array}{l}\text { Kou Valley, } \\
\text { Bukina Faso }\end{array}$ & Netprotect ${ }^{\circledR}$ & Deltamethrin & Incorporation & $\begin{array}{l}10-13 \\
\text { December, } \\
2007\end{array}$ & $\begin{array}{l}\text { An. gambiae } \\
\text { (Kisumu) }\end{array}$ & 100 & 100 \\
\hline WHO, 2008 [12] & $\begin{array}{l}\text { Kou Valley, } \\
\text { Bukina Faso }\end{array}$ & DuraNet ${ }^{\circledR}$ & $\begin{array}{l}\text { Alpha- } \\
\text { cypermethrin }\end{array}$ & Incorporation & $\begin{array}{l}10-13 \\
\text { December, } \\
2007\end{array}$ & $\begin{array}{l}\text { An. gambiae } \\
\text { (Kisumu) }\end{array}$ & 100 & 100 \\
\hline WHO, 2008 [12] & $\begin{array}{l}\text { WHOPES } \\
\text { supervised } \\
\text { studies }\end{array}$ & DuraNet ${ }^{\circledR}$ & $\begin{array}{l}\text { Alpha- } \\
\text { cypermethrin }\end{array}$ & Incorporation & $\begin{array}{l}10-13 \\
\text { December, } \\
2007\end{array}$ & $\begin{array}{l}\text { An. gambiae } \\
\text { (Kisumu) }\end{array}$ & 100 & 98 \\
\hline WHO, 2008 [12] & $\begin{array}{l}\text { Muheza, } \\
\text { Tanzania }\end{array}$ & DuraNet ${ }^{\circledR}$ & $\begin{array}{l}\text { Alpha- } \\
\text { cypermethrin }\end{array}$ & Incorporation & $\begin{array}{l}10-13 \\
\text { December, } \\
2007\end{array}$ & $\begin{array}{l}\text { An. gambiae } \\
\text { (Kisumu) }\end{array}$ & 100 & 100 \\
\hline WHO, 2009 [75] & $\begin{array}{l}\text { Melanville, } \\
\text { North Benin }\end{array}$ & $\begin{array}{l}\text { PermaNet }{ }^{\circledR} \\
2.5\end{array}$ & Deltamethrin & Impregnation & $\begin{array}{l}\text { 8-11 } \\
\text { December, } \\
2008\end{array}$ & $\begin{array}{l}\text { An. gambiae } \\
\text { (Kisumu) }\end{array}$ & 100 & 100 \\
\hline WHO, 2009 [75] & $\begin{array}{l}\text { Melanville, } \\
\text { North Benin }\end{array}$ & $\begin{array}{l}\text { PermaNet }{ }^{\circledR} \\
2.0\end{array}$ & Deltamethrin & Impregnation & $\begin{array}{l}\text { 8-11 } \\
\text { December, } \\
2008\end{array}$ & $\begin{array}{l}\text { An. gambiae } \\
\text { (Kisumu) }\end{array}$ & 100 & 100 \\
\hline WHO, 2009 [75] & $\begin{array}{l}\text { Kilimanjaro } \\
\text { district, } \\
\text { Tanzania }\end{array}$ & $\begin{array}{l}\text { PermaNet® } \\
2.5\end{array}$ & Deltamethrin & Impregnation & $\begin{array}{l}\text { 8-11 } \\
\text { December, } \\
2008\end{array}$ & $\begin{array}{l}\text { An. gambiae } \\
\text { (Kisumu) }\end{array}$ & 100 & 100 \\
\hline WHO, 2009 [75] & $\begin{array}{l}\text { Kilimanjaro } \\
\text { district, } \\
\text { Tanzania }\end{array}$ & $\begin{array}{l}\text { PermaNet }{ }^{\circledR} \\
2.0\end{array}$ & Deltamethrin & Impregnation & $\begin{array}{l}\text { 8-11 } \\
\text { December, } \\
2008\end{array}$ & $\begin{array}{l}\text { An. gambiae } \\
\text { (Kisumu) }\end{array}$ & 100 & 100 \\
\hline
\end{tabular}

* Include new unused, old unused or unwashed Long-Lasting Insecticidal Nets; "Many reviewed studies did not report ITNs manufactured date/year, hence in this review encompass and report either manufactured year/distributed year/tested year or date of meeting; KD60 knockdown measured at 60 minutes (sublethal incapacitation); M24 mortality measured at 24 hours post exposure. 


\begin{tabular}{|c|c|c|c|c|c|c|c|c|}
\hline Author/Report & Location & $\begin{array}{l}\text { Pyrethroid } \\
\text { ITN }^{*}\end{array}$ & $\begin{array}{l}\text { Active } \\
\text { Ingredients }\end{array}$ & $\begin{array}{l}\text { Production } \\
\text { technology }\end{array}$ & Year" & $\begin{array}{l}\text { Mosquito strains } \\
\text { (Susceptible) }\end{array}$ & KD60 & M24 \\
\hline WHO, 2010 [15] & $\begin{array}{l}\text { Montipellier, } \\
\text { France }\end{array}$ & Yorkool® & Deltamethrin & Impregnation & $\begin{array}{l}28-30 \\
\text { July, } 2009\end{array}$ & $\begin{array}{l}\text { An. gambiae } \\
\text { (Kisumu) }\end{array}$ & 100 & 55 \\
\hline WHO, 2010 [15] & $\begin{array}{l}\text { Montipellier, } \\
\text { France }\end{array}$ & $\begin{array}{l}\text { PermaNet }{ }^{\circledR} \\
2.0\end{array}$ & Deltamethrin & Impregnation & $\begin{array}{l}28-30 \\
\text { July, } 2009\end{array}$ & $\begin{array}{l}\text { An. gambiae } \\
\text { (Kisumu) }\end{array}$ & 100 & 100 \\
\hline WHO, 2010 [15] & $\begin{array}{l}\text { Malanville, } \\
\text { Benin }\end{array}$ & DawaPlus ${ }^{\circledR}$ & Deltamethrin & Impregnation & $\begin{array}{l}28-30 \\
\text { July, } 2009\end{array}$ & $\begin{array}{l}\text { An. gambiae } \\
\text { (Kisumu) }\end{array}$ & 100 & 100 \\
\hline WHO, 2010 [15] & $\begin{array}{l}\text { Malanville, } \\
\text { Benin }\end{array}$ & $\begin{array}{l}\text { DawaPlus }{ }^{\circledR} \\
2.0\end{array}$ & Deltamethrin & Impregnation & $\begin{array}{l}28-30 \\
\text { July, } 2009\end{array}$ & $\begin{array}{l}\text { An. gambiae } \\
\text { (Kisumu) }\end{array}$ & 100 & 100 \\
\hline WHO, 2010 [15] & $\begin{array}{l}\text { Muheza, } \\
\text { Tanzania }\end{array}$ & $\begin{array}{l}\text { DawaPlus }{ }^{\circledR} \\
2.0\end{array}$ & Deltamethrin & Impregnation & $\begin{array}{l}28-30 \\
\text { July, } 2009\end{array}$ & $\begin{array}{l}\text { An. gambiae } \\
\text { (Kisumu) }\end{array}$ & 100 & 100 \\
\hline WHO, 2011 [76] & $\begin{array}{l}\text { Montipellier, } \\
\text { France }\end{array}$ & Yahe ${ }^{\circledR}$ & Deltamethrin & Impregnation & $\begin{array}{l}11-15 \\
\text { April, } \\
2011\end{array}$ & $\begin{array}{l}\text { An. gambiae } \\
\text { (Kisumu) }\end{array}$ & 100 & 100 \\
\hline WHO, 2011 [76] & $\begin{array}{l}\text { Montipellier, } \\
\text { France }\end{array}$ & $\begin{array}{l}\text { PermaNet }{ }^{\circledR} \\
2.0\end{array}$ & Deltamethrin & Impregnation & $\begin{array}{l}11-15 \\
\text { April, } \\
2011\end{array}$ & $\begin{array}{l}\text { An. gambiae } \\
\text { (Kisumu) }\end{array}$ & 100 & 100 \\
\hline WHO, 2011 [76] & $\begin{array}{l}\text { Montipellier, } \\
\text { France }\end{array}$ & $\begin{array}{l}\text { Royal } \\
\text { Sentry }{ }^{\circledR}\end{array}$ & $\begin{array}{l}\text { Alpha- } \\
\text { cypermethrin }\end{array}$ & Incorporation & $\begin{array}{l}11-15 \\
\text { April, } \\
2011\end{array}$ & $\begin{array}{l}\text { An. gambiae } \\
\text { (Kisumu) }\end{array}$ & 100 & 100 \\
\hline WHO, 2011 [76] & $\begin{array}{l}\text { Montipellier, } \\
\text { France }\end{array}$ & DuraNet ${ }^{\circledR}$ & $\begin{array}{l}\text { Alpha- } \\
\text { cypermethrin }\end{array}$ & Incorporation & $\begin{array}{l}11-15 \\
\text { April, } \\
2011\end{array}$ & $\begin{array}{l}\text { An. gambiae } \\
\text { (Kisumu) }\end{array}$ & 100 & 100 \\
\hline WHO, 2011 [76] & $\begin{array}{l}\text { Montipellier, } \\
\text { France }\end{array}$ & MagNet ${ }^{\circledR}$ & $\begin{array}{l}\text { Alpha- } \\
\text { cypermethrin }\end{array}$ & Incorporation & $\begin{array}{l}11-15 \\
\text { April, } \\
2011\end{array}$ & $\begin{array}{l}\text { An. gambiae } \\
\text { (Kisumu) }\end{array}$ & 100 & 100 \\
\hline WHO, 2011 [76] & & LifeNet ${ }^{\circledR}$ & Deltamethrin & Incorporation & $\begin{array}{l}11-15 \\
\text { April, } \\
2011\end{array}$ & $\begin{array}{l}\text { An. gambiae } \\
\text { (Kisumu) }\end{array}$ & 100 & 100 \\
\hline WHO, 2012 [77] & India & Interceptor® & $\begin{array}{l}\text { Alpha- } \\
\text { cypermethrin }\end{array}$ & Impregnation & $\begin{array}{l}18-22 \\
\text { June, } \\
2012\end{array}$ & An. culicifacies & 97.8 & 98 \\
\hline WHO, 2012 [77] & $\begin{array}{l}\text { Muheza, } \\
\text { Tanzania }\end{array}$ & Interceptor® & $\begin{array}{l}\text { Alpha- } \\
\text { cypermethrin }\end{array}$ & Impregnation & $\begin{array}{l}18-22 \\
\text { June, } \\
2012\end{array}$ & $\begin{array}{l}\text { An. gambiae } \\
\text { (Kisumu) }\end{array}$ & 100 & 99 \\
\hline WHO, 2012 [77] & Benin & Olyset $\mathrm{Net}{ }^{\circledR}$ & Permethrin & Incorporation & $\begin{array}{l}18-22 \\
\text { June, } \\
2012\end{array}$ & $\begin{array}{l}\text { An. gambiae } \\
\text { (Kisumu) }\end{array}$ & 100 & 37 \\
\hline WHO, 2013 [78] & $\begin{array}{l}\text { Mae Sot } \\
\text { District, } \\
\text { Thailand }\end{array}$ & $\begin{array}{l}\text { PermaNet }{ }^{\circledR} \\
2.0\end{array}$ & Deltamethrin & Impregnation & $\begin{array}{l}22-30 \\
\text { July, } 2013\end{array}$ & An. culicifacies & 100 & 100 \\
\hline WHO, 2013 [78] & $\begin{array}{l}\text { Muheza, } \\
\text { Tanzania }\end{array}$ & Yahe ${ }^{\circledR}$ & Deltamethrin & Impregnation & $\begin{array}{l}22-30 \\
\text { July, } 2013\end{array}$ & $\begin{array}{l}\text { An. gambiae } \\
\text { (Kisumu) }\end{array}$ & 100 & 100 \\
\hline WHO, 2013 [78] & $\begin{array}{l}\text { Mae Sot } \\
\text { District, } \\
\text { Thailand }\end{array}$ & Yahe® & Deltamethrin & Impregnation & $\begin{array}{l}22-30 \\
\text { July, } 2013\end{array}$ & An. minimus & 73 & 58 \\
\hline WHO, 2013 [78] & $\begin{array}{l}\text { Muheza, } \\
\text { Tanzania }\end{array}$ & $\begin{array}{l}\text { PermaNet }{ }^{\circledR} \\
2.0\end{array}$ & Deltamethrin & Impregnation & $\begin{array}{l}22-30 \\
\text { July, } 2013\end{array}$ & $\begin{array}{l}\text { An. gambiae } \\
\text { (Kisumu) }\end{array}$ & 100 & 100 \\
\hline WHO, 2013 [78] & $\begin{array}{l}\text { Rourkela, } \\
\text { India }\end{array}$ & DuraNet ${ }^{\circledR}$ & $\begin{array}{l}\text { Alpha- } \\
\text { cypermethrin }\end{array}$ & Incorporation & $\begin{array}{l}22-30 \\
\text { July, } 2013\end{array}$ & An. culicifacies & 100 & 100 \\
\hline WHO, 2015 [14] & $\begin{array}{l}\text { WHOPES } \\
\text { supervised } \\
\text { studies }\end{array}$ & Yahe® & Deltamethrin & Impregnation & $\begin{array}{l}29 \text { June-1 } \\
\text { July, } 2015\end{array}$ & $\begin{array}{l}\text { An. gambiae } \\
\text { (Kisumu) }\end{array}$ & 93 & 97 \\
\hline
\end{tabular}

* Include new unused, old unused or unwashed Long-Lasting Insecticidal Nets; ${ }^{\#}$ Many reviewed studies did not report ITNs manufactured date/year, hence in this review encompass and report either manufactured year/distributed year/tested year or date of meeting; KD60 knockdown measured at 60 minutes (sublethal incapacitation); M24 mortality measured at 24 hours post exposure. 


\begin{tabular}{|c|c|c|c|c|c|c|c|c|}
\hline Author/Report & Location & $\begin{array}{l}\text { Pyrethroid } \\
\text { ITN* }^{*}\end{array}$ & $\begin{array}{l}\text { Active } \\
\text { Ingredients }\end{array}$ & $\begin{array}{l}\text { Production } \\
\text { technology }\end{array}$ & Year"\# & $\begin{array}{l}\text { Mosquito strains } \\
\text { (Susceptible) }\end{array}$ & KD60 & M24 \\
\hline WHO, 2015 [14] & $\begin{array}{l}\text { Montipellier, } \\
\text { France }\end{array}$ & SafeNet® & $\begin{array}{l}\text { Alpha- } \\
\text { cypermethrin }\end{array}$ & Impregnation & $\begin{array}{l}29 \text { June-1 } \\
\text { July, } 2015\end{array}$ & $\begin{array}{l}\text { An. gambiae } \\
\text { (Kisumu) }\end{array}$ & 100 & 99.5 \\
\hline WHO, 2015 [14] & $\begin{array}{l}\text { Montipellier, } \\
\text { France }\end{array}$ & SafeNet® & $\begin{array}{l}\text { Alpha- } \\
\text { cypermethrin }\end{array}$ & Impregnation & $\begin{array}{l}29 \text { June-1 } \\
\text { July, } 2015\end{array}$ & $\begin{array}{l}\text { An. gambiae } \\
\text { (Kisumu) }\end{array}$ & 100 & 100 \\
\hline WHO, 2015 [14] & $\begin{array}{l}\text { Montipellier, } \\
\text { France }\end{array}$ & Interceptor ${ }^{\circledR}$ & $\begin{array}{l}\text { Alpha- } \\
\text { cypermethrin }\end{array}$ & Impregnation & $\begin{array}{l}29 \text { June-1 } \\
\text { July, } 2015\end{array}$ & $\begin{array}{l}\text { An. gambiae } \\
\text { (Kisumu) }\end{array}$ & 100 & 100 \\
\hline WHO, 2015 [14] & $\begin{array}{l}\text { Montipellier, } \\
\text { France }\end{array}$ & $\begin{array}{l}\text { PermaNet }{ }^{\circledR} \\
2.0\end{array}$ & Deltamethrin & Impregnation & $\begin{array}{l}29 \text { June-1 } \\
\text { July, } 2015\end{array}$ & $\begin{array}{l}\text { An. gambiae } \\
\text { (Kisumu) }\end{array}$ & 100 & 100 \\
\hline WHO, 2015 [14] & Côte d'Ivoire & $\begin{array}{l}\text { PermaNet }{ }^{\circledR} \\
2.0\end{array}$ & Deltamethrin & Impregnation & $\begin{array}{l}29 \text { June-1 } \\
\text { July, } 2015\end{array}$ & $\begin{array}{l}\text { An. gambiae } \\
\text { (Kisumu) }\end{array}$ & 100 & 100 \\
\hline WHO, 2015 [14] & $\begin{array}{l}\text { WHOPES } \\
\text { supervised } \\
\text { studies }\end{array}$ & MiraNet巴 & $\begin{array}{l}\text { Alpha- } \\
\text { cypermethrin }\end{array}$ & Incorporation & $\begin{array}{l}29 \text { June- } \\
1 \text { July, } \\
2015\end{array}$ & $\begin{array}{l}\text { An. gambiae } \\
\text { (Kisumu) }\end{array}$ & 100 & 100 \\
\hline WHO, 2015 [14] & Côte d'Ivoire & $\begin{array}{l}\text { Panda } \mathrm{Net} \circledast \\
2.0\end{array}$ & Deltamethrin & Incorporation & $\begin{array}{l}29 \text { June- } \\
1 \text { July, } \\
2015\end{array}$ & $\begin{array}{l}\text { An. gambiae } \\
\text { (Kisumu) }\end{array}$ & 100 & 100 \\
\hline WHO, 2015 [14] & $\begin{array}{l}\text { WHOPES } \\
\text { supervised } \\
\text { studies }\end{array}$ & $\begin{array}{l}\text { Panda Net } \circledast \\
2.0\end{array}$ & Deltamethrin & Incorporation & $\begin{array}{l}29 \text { June- } \\
1 \text { July, } \\
2015\end{array}$ & $\begin{array}{l}\text { An. gambiae } \\
\text { (Kisumu) }\end{array}$ & 97 & 51 \\
\hline WHO, 2019 [79] & $\begin{array}{l}\text { Ifakara } \\
\text { Health } \\
\text { Institute, } \\
\text { Tanzania }\end{array}$ & $\begin{array}{l}\text { Royal } \\
\text { Sentry }{ }^{\circledR} 2.0\end{array}$ & $\begin{array}{l}\text { Alpha- } \\
\text { cypermethrin }\end{array}$ & Incorporation & 2017 & $\begin{array}{l}\text { An. gambiae } \\
\text { (Ifakara strain) }\end{array}$ & 100 & 100 \\
\hline WHO, 2020 [80] & $\begin{array}{l}\text { Reference } \\
\text { laboratory }\end{array}$ & Tsara Soft ${ }^{\circledR}$ & Deltamethrin & Incorporation & 2019 & An. dirus & 95 & 80 \\
\hline
\end{tabular}

\section{Discussion}

The present study explored the utility of cone bioassays for pre-delivery QA of pyrethroid ITNs in two test facilities using different mosquito species to test the assumption that cone bioassays are consistent and reproducible across locations, mosquito strains, and laboratories, and could be conducted in addition to physiochemical tests currently recommended for quality control of ITNs [12]. This study specifically compared the cone bioefficacy results of unused pyrethroid ITNs from PNG in two different test facilities using susceptible An. gambiae s.s and susceptible An. farauti s.s, respectively. Consistency of bioefficacy results between IHI and PNGIMR was demonstrated ( $k=79$ and $90 \%$ accuracy), based on WHO pass/fail criteria, although absolute agreement of cone assay values between IHI and PNGIMR testing facilities was not observed, especially among those nets with low M24.

In this study, the majority of unused pyrethroid ITNs passed efficacy criteria in cone bioassays indicating that the cone bioassay is a sensitive method to identify those nets with sufficient insecticide doses on the net surface to kill and incapacitate pyrethroid susceptible mosquitoes and may provide a means to identify nets with suboptimal insecticide doses on the net surface even with different mosquito species used. Most previous studies using cone bioassay tests reported bioefficacy above WHO critical thresholds for unused pyrethroid ITNs [14-25]. However, some studies reported bioefficacy below WHO critical thresholds in Benin [38], Iran [39], Madagascar [40] and PNG [41]. The reasons for this are unclear but our study corroborates the recent findings from PNG [41]. However, it is known that cone bioassay results can be affected by ITN characteristics i.e. manufacturing processes [42-44], poor shipping [45] or storage conditions [46]; as well as bioassay methods including sample preparation e.g. using a net sample straight from the fridge (Skovmand, personal communication), mosquito age $[47,48]$ and fitness [49]), test procedures [36], temperature [50-52] and inter-operator variability [12].

In this study, five of the twenty unused ITNs effectively killed mosquitoes ( $\geq 80 \% \mathrm{M} 24)$ at IHI and the best performing unused ITNs had mean M24 of $99 \%$ and the worst performing unused ITNs had mean M24 of $24 \%$ measured in cone bioassay test (average from the two sites). These results agree well with other studies and WHO specification reports [15, 33, 38-41]..Even so,, most ITNs tested at IHI gave higher KD60 than M24. It has been observed that with An. gambiae to achieve $80 \%$ M24 requires at least a 5\% higher net surface concentration of pyrethroid than to achieve 95\% KD60 [12]. It was reported in an expert review that new unused nets demonstrate 100\% KD60 but 55\% M24 [15]. It may 
therefore be inferred that M24 is the more conservative endpoint and the best benchmark of pyrethroid performance in a cone bioassay. When M24 of 50\% was used as the threshold for passing, the cone test still agreed well between the two sites, suggesting that M24 may be a more optimal endpoint to use for quality control, as historically suggested [53]. Indeed, it was previously stipulated by WHOPES that "As the two existing WHO criteria for biological effect in the cone test correspond to different surface concentrations of active ingredient, they are not equivalent and one them should be designated as the basis for WHO specifications. Possibly the criterion could be chosen on a case-by-case basis but mortality is clearly more stringent than KD and therefore appears to be the criterion of choice"[30]. However, all nets passed tunnel tests, possibly because of longer mosquitoes exposure time (12 hours) compared to cone assay test ( 3 minutes) as well as sugar starvation in the tunnel test [8], that gives higher efficacy even at lower pyrethroid concentrations [20,54]. Based on the results of the literature review, that the need for tunnel tests for predicting new pyrethroid ITN bioefficacy is questionable. All analyses conducted showed greater agreement between the two sites when 24-hour mortality was used as the endpoint. Spearman correlation showed very strong correlation of efficacy results for M24 ( $r=0.9$ ) between the two testing facilities (Figure 3) and the Bland Altman showed more consistent agreement on this endpoint. These results further corroborate other confirmatory analyses of PNGIMR bioefficacy tests conducted in Liverpool School of Tropical Medicine (LSTM) where susceptible An. gambiae s.s. mortality estimates were strongly correlated with PNGIMR results (coefficient of determination equal to 0.80) [41].

In some countries with high malaria burden, e.g. Nigeria, ITN quality control using a bioassay test was utilized after a long period of importation of nets with low bioefficacy [55]. It has also been hypothesized that the provision of lower efficacy nets has contributed to a significant rise of malaria cases in some high malaria burden countries including Nicaragua [56], Nigeria [55], PNG [41], Rwanda [57], Solomon of Islands [58]. Acceptable performance of ITNs is defined by WHO as retention of biological activity (e.g. mosquito mortality $\geq 80 \%$ ) through 20 standard washes (or 3 years of use) but there is no simple physiochemical measurement corresponding to this definition [30]. Mosquitoes which land on the netting are exposed only to active ingredient on the surface but surface concentrations are practically difficult to measure. Moreover, a recent study reported unused new nets with acceptable product specifications i.e. chemical content did not effectively kill or knockdown mosquitoes in cone bioassay test[41].

The present study demonstrates a high rate of agreement between cone bioassay results for paired net samples and only a few nets had discrepant results between IHI and PNGIMR testing facilities. This is particularly true if assay-inherent stochastic variability is considered. However, overall results show higher knockdown rate and low mortality rate at IHI compared to PNGIMR and discrepant results obtained for two nets which is likely to be due to random errors and/or systematic bias in studies. This can be seen in the large error bars around the mean value of mortality for these ITNs in the PNGIMR and higher knockdown rate in IHI (Figure 5).

Some of the observed differences are likely to be due to testing conditions and procedures at two facilities. Differences that cannot be ruled out are temperature that is known to impact mortality $[51,52]$. The temperature in PNG was $28 \pm 4^{\circ} \mathrm{C}$ compared to $27 \pm 1^{\circ} \mathrm{C}$ at IHI, although control mortality was acceptable at both sites. Variability in cone bioassay procedures i.e. the angle of cone was $45^{\circ}$ [41] as per WHO guidelines in PNGIMR, while in IHI the cone test is performed at $60^{\circ}$ in the cone assay board to maximise mosquito contact with ITNs although this has been shown to be inconsequential [36]. Net pieces were shipped to Tanzania from PNG by courier in an insulated package with very short transit time. As such it is unlikely that transport would have affected their bioefficacy. Also, it is unlikely that operator skill contributed variability of results, because repeated cone tests conducted on the same pieces at different time points gave similar results. The An. gambiae s.s and An. farauti s.s strains used were fully susceptible to pyrethroid insecticides, of similar age and exposed to similar colony maintenance conditions; these strains are not sibling species and they have differing morphology [59]. The strain used for this test has shown high mortality in cone tests against several ITN brands including the ones tested in this current study [46, 60]. It is currently unclear how susceptible mosquito species affects the outcome of cone bioassays and more research is needed to establish robust parameters for comparison. Mosquitoes species variability e.g. size, cuticular thickness may explain some of the variation in absolute values measured, and similar differences have been observed in other multi-center studies determining the bioefficacy of the same nets [33]. Even so, the cone bioassay showed very good agreement which was highly related for those nets that demonstrated highest M24. Unsurprisingly, more variation in results was observed between the testing facilities for ITNs with low knockdown or mortality. This is a well-known phenomenon and for this reason large sample sizes (30-50 nets) are recommended for cone testing used for bioefficacy monitoring of field used ITNs that generally have reduced M24 [8].

\section{Study Limitations}

The number of nets tested may not be sufficient to generalize the study results; therefore, a review of literature on cone bioassay bioefficacy evaluation of unused pyrethroid nets was also conducted. These data also demonstrate that almost all new or unused pyrethroid ITNs with data available in the published literature demonstrated excellent KD60 and M24. While it is highly likely that there would be a bias toward publication of positive trials [61] no difference in the average knockdown or mortality values between the WHO reports or published literature was observed. Even so, the authors feel that it is critical that WHO resumes reporting ITN performance data in prequalification reports to be used as a product performance reference by procurement agencies, National Malaria Control Programs (NMCP) or other bodies that monitor product performance at a country level. Also of note, many publications and reports did not indicate country of manufacture, ITN age, and

Page $14 / 25$ 
lot/batch of the tested nets or data collection period. This information is useful to aid procurement agencies or manufacturers to investigate any possible product failures and pinpoint probable causes such as poor shipping, storage or batch variability.

\section{Conclusions}

Based on these study findings, the WHO cone test is a highly reproducible bioassay that can be used as a reliable indicator of ITN quality. The literature review included in this study confirms that WHO standard efficacy criteria in cone bioassays have been previously achieved by all pyrethroid ITNs (unwashed), without the need for additional tunnel tests. The use of combined knockdown and mortality endpoint gives more consistent agreement than using either KD60 or M24 endpoint alone. However, the $80 \%$ M24 threshold remains the most reliable indicator of pyrethroid ITN quality among pyrethroid susceptible mosquitoes. As part of pre-delivery QA, cone tests could assess the availability of active ingredients at the surface of the net where mosquitoes encounter it.

\section{Abbreviations}

Al

Active ingredient

$\mathrm{Cl}$

Confidence interval

IHI

Ifakara Health Institute

IMR

Institute of Medical Research

ITNs

Insecticide treated nets

LLINs

Long-lasting insecticidal treated nets

KD60

Knockdown after 60-minutes:M24:Mortality at 24-hours

PNG

Papua New Guinea

QA

Quality Assurance

VCPTU

Vector Control Product Testing Unit

WHO

World Health Organization.

\section{Declarations}

\section{Acknowledgment}

The authors thank everyone who supported this study either directly or indirectly including the entire VCPTU team at IHI, Tanzania and the PNG National Malaria Control Program, especially Mr. Leo Makita, and Rotarians Against Malaria PNG for their support.

\section{Author's contribution}

SJM, TF and SK conceived the study. SGM conducted the study in IHI, Tanzania; NB and SK conducted study in PNGIMR; EKM, JM, KM assisted in experiment setting and data collection; SGM performed data analysis; SJM and SK guided data analysis plan; SGM wrote the manuscript; DK, ML, EKM, OGO, TF read and reviewed the manuscript; SJM, EM and SK critically revised the final manuscript; SJM and EM supervised the study.

\section{Funding}

The study was funded in Tanzania by Vector Control Product Testing Unit (VCPTU) and the Training and Capacity Building Unit of Ifakara Health Institute (IHI). Work in Papua New Guinea Institute of Medical Research was supported by a National Health and Medical Research 


\section{Availability of data and materials}

The data set for this study is available on request from VCPTU.

\section{Ethics approval and consent to participate}

Approval for the study was provided by the Institutional Review board of Ifakara Health Institute (IHI/IRB/No: 23-202) and National Institute of Medical Research, PNGIMR Internal Review Board and the PNG Medical Research Advisory Committee (MRAC 21.02). This study did not involve humans as study participants.

\section{Consent for publication}

Not applicable.

\section{Competing Interests}

The authors declare that they have no competing interests. SJM, EM, JM, KM, OGO evaluate vector control products including ITNs for a number of manufacturers including the manufacturers of several ITNs reported in this article. No manufacturers of ITNs had any input into the article.

\section{Author details}

${ }^{1}$ Vector Control Product Testing Unit (VCPTU), Environmental Health and Ecological Science Department, Ifakara Health Institute, Tanzania. ${ }^{2}$ Nelson Mandela Africa Institution of Science and Technology, Arusha, Tanzania. ${ }^{3}$ Sokoine RRH, Ministry of Health, Tanzania. ${ }^{4}$ Regional Health Management Team, P.O Box 1011, Lindi, Southern Tanzania. ${ }^{5}$ Vector borne Disease Unit, PNG Institute of Medical Research, Madang, P.O Box 378, Madang Province 511, Papua New Guinea. ${ }^{6}$ University of Basel, Basel, Switzerland. ${ }^{7}$ Swiss Tropical and Public Health Institute, Basel, Switzerland. ${ }^{8}$ London School of Hygiene and Tropical Medicine, London. ${ }^{9}$ Rotarian Against Malaria. P.O Box 3686 Boroko NCD 111 Papua New Guinea. ${ }^{10}$ Australian Institute of Tropical Health and Medicine, James Cook University, 1/14-88 McGregor Road, Smithfield QLD 4870 Australia.

\section{References}

1. WHO. The guideline for Malaria. World Health Organization; 2021.

2. Bhatt S, Weiss D, Cameron E, Bisanzio D, Mappin B, Dalrymple U, Battle K, Moyes C, Henry A, Eckhoff P. The effect of malaria control on Plasmodium falciparum in Africa between 2000 and 2015. Nature. 2015;526:207-11.

3. WHO. Global report on insecticide resistance in malaria vectors. World Health Organization; 2018.

4. Sherrard-Smith E, Skarp JE, Beale AD, Fornadel C, Norris LC, Moore SJ, Mihreteab S, Charlwood JD, Bhatt S, Winskill P, et al: Mosquito feeding behavior and how it influences residual malaria transmission across Africa. Proceedings of the National Academy of Sciences 2019:201820646.

5. Kleinschmidt I, Bradley J, Knox TB, Mnzava AP, Kafy HT, Mbogo C, Ismail BA, Bigoga JD, Adechoubou A, Raghavendra K, et al. Implications of insecticide resistance for malaria vector control with long-lasting insecticidal nets: a WHO-coordinated, prospective, international, observational cohort study. Lancet Infect Dis. 2018;18:640-9.

6. Koimbu G, Czeher C, Katusele M, Sakur M, Kilepak L, Tandrapah A, Hetzel MW, Pulford J, Robinson L, Karl S. Status of insecticide resistance in Papua New Guinea: an update from nation-wide monitoring of Anopheles mosquitoes. Am J Trop Med Hyg. 2018;98:162.

7. Lindsay SW, Thomas MB, Kleinschmidt I. Threats to the effectiveness of insecticide-treated bednets for malaria control: thinking beyond insecticide resistance. The Lancet Global Health 2021.

8. WHO. Guidelines for laboratory and field-testing of long-lasting insecticidal nets. World Health Organization; 2013.

9. WHOPES. Guidelines for laboratory and field testing of long-lasting insecticidal mosquito nets WHO/CDS/WHOPES/GCDPP/2005.11. Geneva: World Health Organization Pesticide Evaluation Scheme; 2005. 
10. Hawley WA, Phillips-Howard PA, ter Kuile FO, Terlouw DJ, Vulule JM, Ombok M, Nahlen BL, Gimnig JE, Kariuki SK, Kolczak MS. Community-wide effects of permethrin-treated bed nets on child mortality and malaria morbidity in western Kenya. Am J Trop Med Hyg. 2003;68:121-7.

11. Skovmand O, Dang DM, Tran TQ, Bossellman R, Moore SJ. From the factory to the field: considerations of product characteristics for insecticide-treated net (ITN) bioefficacy testing. Malaria Journal. 2021;20:1-13.

12. WHO. Report of the eleventh WHOPES working group meeting. 2008.

13. WHO. Guidelines for laboratory and field testing of long-lasting insecticidal mosquito nets. World Health Organization; 2005.

14. WHO. Report of the eighteenth WHOPES working group meeting: WHO/HQ, Geneva. World Health Organization; 2015.

15. WHO. Report of the thirteenth [13th] WHOPES working group meeting: WHO/HQ, Geneva. World Health Organization; 2009.

16. Okia M, Ndyomugyenyi R, Kirunda J, Byaruhanga A, Adibaku S, Lwamafa DK, Kironde F. Bioefficacy of long-lasting insecticidal nets against pyrethroid-resistant populations of Anopheles gambiae ss from different malaria transmission zones in Uganda. Parasites vectors. 2013;6:1-10.

17. Graham K, Kayedi M, Maxwell C, Kaur H, Rehman H, Malima R, Curtis C, Lines J, Rowland M. Multi-country field trials comparing washresistance of PermaNet ${ }^{\mathrm{TM}}$ and conventional insecticide-treated nets against anopheline and culicine mosquitoes. Med Vet Entomol. 2005;19:72-83.

18. Castellanos ME, Rodas S, Juárez JG, Lol JC, Chanquin S, Morales Z, Vizcaino L, Smith SC, Eng JV, Woldu HG. Evaluation of the durability of long-lasting insecticidal nets in Guatemala. Malar J. 2021;20:1-14.

19. Tungu PK, Michael E, Sudi W, Kisinza WW, Rowland M. Efficacy of interceptor® G2, a long-lasting insecticide mixture net treated with chlorfenapyr and alpha-cypermethrin against Anopheles funestus: experimental hut trials in north-eastern Tanzania. Malaria Journal. $2021 ; 20: 1-15$.

20. Rafinejad J, Vatandoost H, Nikpoor F, Abai M, Shaeghi M, Duchen S, Rafi F. Effect of washing on the bio-efficacy of insecticide-treated nets (ITNs) and long-lasting insecticidal nets (LLINs) against main malaria vector Anopheles stephensi by three bioassay methods. $J$ Vector Borne Dis. 2008;45:143.

21. Abílio AP, Marrune P, de Deus N, Mbofana F, Muianga P, Kampango A. Bio-efficacy of new long-lasting insecticide-treated bed nets against Anopheles funestus and Anopheles gambiae from central and northern Mozambique. Malar J. 2015;14:1-15.

22. Malima R, Tungu PK, Mwingira V, Maxwell C, Magesa SM, Kaur H, Kirby MJ, Rowland M. Evaluation of the long-lasting insecticidal net Interceptor LN: laboratory and experimental hut studies against anopheline and culicine mosquitoes in northeastern Tanzania. Parasites vectors. 2013;6:1-11.

23. Bhatt RM, Sharma SN, Uragayala S, Dash AP, Kamaraju R. Effectiveness and durability of Interceptor® long-lasting insecticidal nets in a malaria endemic area of central India. Malar J. 2012;11:1-8.

24. Ketoh GK, Ahadji-Dabla KM, Chabi J, Amoudji AD, Apetogbo GY, Awokou F, Glitho IA. Efficacy of two PBO long lasting insecticidal nets against natural populations of Anopheles gambiae s. I. in experimental huts, Kolokopé, Togo. PloS one. 2018;13:e0192492.

25. Kayedi M, Lines J, Haghdoost A, Behrahi A, Khamisabadi K. Entomological evaluation of three brands of manufactured insecticidal nets and of nets conventionally treated with deltamethrin, after repeated washing. Annals of Tropical Medicine Parasitology. 2007;101:449-56.

26. Massue DJ, Lorenz LM, Moore JD, Ntabaliba WS, Ackerman S, Mboma ZM, Kisinza WN, Mbuba E, Mmbaga S, Bradley J. Comparing the new Ifakara Ambient Chamber Test with WHO cone and tunnel tests for bioefficacy and non-inferiority testing of insecticide-treated nets. Malar J. 2019;18:1-15.

27. Gimnig JE, Lindblade KA, Mount DL, Atieli FK, Crawford S, Wolkon A, Hawley WA, Dotson EM. Laboratory wash resistance of long-lasting insecticidal nets. Tropical Med Int Health. 2005;10:1022-9.

28. Global Fund. Guide to Global Fund Policies on Procurement and Supply Management of Health Products. Geneva: Global Fund; 2018.

29. Th LLP. Landscaping of ITN Bioefficacy Report for The Global Fund https://www.trophealth.com/wp-content/uploads/2021/12/ITNBioefficacy-Landscaping-Final-Report.pdf. Tropical health LLP; 2021.

30. WHOPES. Report of the eleventh WHOPES working group meeting: WHO/HQ, Geneva, 10-13 December 2006 : review of Spinosad 7.48\% DT, Netprotect, Duranet, Dawaplus, Icon Maxx. Geneva: World Health Organization; 2006.

31. Karl S, Katusele M, Freeman TW, Moore SJ. Quality control of long-lasting insecticidal nets: are we neglecting it? Trends in Parasitology 2021.

32. Villalta EL, Soto Bravo AM, Vizcaino L, Dzuris N, Delgado M, Green M, Smith SC, Lenhart A. Macedo de Oliveira A: Evaluation of the durability and use of long-lasting insecticidal nets in Nicaragua. Malaria Journal. 2021;20:106.

33. WHO. Report of the tenth WHOPES working group meeting: WHO/HQ Geneva. World Health Organization; 2007.

34. SANAS. Good Laboratory Practice compliant facilities. 2021.

Page $17 / 25$ 
35. MR4. Methods in Anopheles Research Manual https://www.beiresources.org/Publications/MethodsinAnophelesResearch.aspx 2009.

36. Owusu HF, Müller P. How important is the angle of tilt in the WHO cone bioassay? Malaria Journal. 2016;15:1-10.

37. Bland JM, Altman DG: Measuring agreement in method comparison studies. Stat Methods Med Res 1999 8: $1351999,8: 135-160$.

38. Ahogni I, Aïkpon R, Ossè R, Dagnon J, Govoetchan R, Attolou R, Agbevo A, Azondekon R, Koukpo C, Gnanguenon V. Field durability of Yorkool ${ }^{\circledR}$ LN nets in the Benin Republic. Advances in Entomology. 2019;8:72-92.

39. Bagheri A, Vatandoost H, Shayeghi M, Abai MR, Raeisi A, Godwin NG, Akbari M, Sheikhi S. Evaluation on the bioefficacy of PermaNet ${ }^{\circledR} 2.0$, a long lasting net against Anopheles stephensi. Asian Pac J Trop Dis. 2017;7:775-7.

40. Randriamaherijaona S, Raharinjatovo J, Boyer S. Durability monitoring of long-lasting insecticidal (mosquito) nets (LLINs) in Madagascar: physical integrity and insecticidal activity. Parasites vectors. 2017;10:1-11.

41. Vinit R, Timinao L, Bubun N, Katusele M, Robinson LJ, Kaman P, Sakur M, Makita L, Reimer L, Schofield L. Decreased bioefficacy of longlasting insecticidal nets and the resurgence of malaria in Papua New Guinea. Nature communications. 2020;11:1-7.

42. Müller O, Ido K, Traore C. Evaluation of a prototype long-lasting insecticide-treated mosquito net under field conditions in rural Burkina Faso. Trans R Soc Trop Med Hyg. 2002;96:483-4.

43. Kilian A, Byamukama W, Pigeon O, Atieli F, Duchon S, Phan C. Long-term field performance of a polyester-based long-lasting insecticidal mosquito net in rural Uganda. Malar J. 2008;7:1-22.

44. Skovmand O, Dang DM, Tran TQ, Bossellman R, Moore SJ. From the factory to the field: considerations of product characteristics for insecticide-treated net (ITN) bioefficacy testing. Malar J. 2021;20:363.

45. AMP. Use of containers to store insecticide-treated nets: operational concerns and considerations. The Alliance for Malaria Prevention; 2020.

46. Musa JJ, Moore SJ, Moore J, Mbuba E, Mbeleya E, Kobe D, Swai JK, Odufuwa OG. Long-lasting insecticidal nets retain bio-efficacy after 5 years of storage: implications for malaria control programmes. Mal J 2020, 19.

47. Marti-Soler H, Máquina M, Opiyo M, Alafo C, Sherrard-Smith E, Malheia A, Cuamba N, Sacoor C, Rabinovich R, Aide P, et al. Effect of wall type, delayed mortality and mosquito age on the residual efficacy of a clothianidin-based indoor residual spray formulation (SumiShield ${ }^{\mathrm{TM}}$ 50WG) in southern Mozambique. PLOS ONE. 2021;16:e0248604.

48. Kulma K, Saddler A, Koella JC. Effects of age and larval nutrition on phenotypic expression of insecticide-resistance in Anopheles mosquitoes. PLoS One. 2013;8:e58322.

49. Owusu HF, Chitnis N, Müller P. Insecticide susceptibility of Anopheles mosquitoes changes in response to variations in the larval environment. Sci Rep. 2017;7:3667.

50. Hodjati MH, Curtis CF. Effects of permethrin at different temperatures on pyrethroid-resistant and susceptible strains of Anopheles. Med Vet Entomol. 1999;13:415-22.

51. Glunt KD, Oliver SV, Hunt R, Paaijmans KP. The impact of temperature on insecticide toxicity against the malaria vectors Anopheles arabiensis and Anopheles funestus. Mal J 2018, 17.

52. Glunt KD, Paaijmans KP, Read AF, Thomas MB. Environmental temperatures significantly change the impact of insecticides measured using WHOPES protocols. Malaria Journal. 2014;13:350.

53. WHO. Vector Control Technical Expert Group Report to MPAC September 2013 Estimating functional survival of long-lasting insecticidal nets from field data. Geneva: World Health Organisation; 2013.

54. Hougard J-M, Duchon S, Darriet F, Zaim M, Rogier C, Guillet P. Comparative performances, under laboratory conditions, of seven pyrethroid insecticides used for impregnation of mosquito nets. Bull World Health Organ. 2003;81:324-33.

55. Daniel MI. Quality control of mosquito insecticide treated nets as a preventive measure. J Vector Borne Dis. $2006 ; 43: 92$.

56. Villalta EL, Bravo AMS, Vizcaino L, Dzuris N, Delgado M, Green M, Smith SC, Lenhart A, de Oliveira AM. Evaluation of the durability and use of long-lasting insecticidal nets in Nicaragua. Malaria Journal. 2021;20:1-13.

57. Karema C, Wen S, Sidibe A, Smith JL, Gosling R, Hakizimana E, Tanner M, Noor AM, Tatarsky A. History of malaria control in Rwanda: implications for future elimination in Rwanda and other malaria-endemic countries. Malar J. 2020;19:1-12.

58. WHO. Ten years in public health: report by Dr Margaret Chan, Director-General. World Health Organization; 2017.

59. Manguin S. Anopheles mosquitoes: new insights into malaria vectors. BoD-Books on Demand; 2013.

60. Lorenz LM, Bradley J, Yukich J, Massue DJ, Mageni Mboma Z, Pigeon O, Moore J, Kilian A, Lines J, Kisinza W, et al. Comparative functional survival and equivalent annual cost of 3 long-lasting insecticidal net (LLIN) products in Tanzania: A randomised trial with 3year follow up. PLOS Medicine. 2020;17:e1003248.

61. Kennedy D. The old file-drawer problem. Science. 2004;305:451.

Page $18 / 25$ 
62. Agossa FR, Padonou GG, Gnanguenon V, Oké-Agbo F, Zola-Sahossi J, Dègnonvi H, Salako A, Sèzonlin M, Akogbéto MC. Laboratory and field evaluation of the impact of washings on the effectiveness of LifeNet ${ }^{\circledR}$, Olyset ${ }^{\circledR}$ and PermaNet ${ }^{\circledR} 2.0$ in two areas, where there is a high level of resistance of Anopheles gambiae to pyrethroids, Benin, West Africa. Malaria Journal. 2014;13:1-10.

63. Allossogbe M, Gnanguenon V, Yovogan B, Akinro B, Anagonou R, Agossa F, Houtoukpe A, Padonou GG, Akogbeto M. WHO cone bio-assays of classical and new-generation long-lasting insecticidal nets call for innovative insecticides targeting the knock-down resistance mechanism in Benin. Malaria Journal. 2017;16:1-11.

64. Camara S, Alou LPA, Koffi AA, Clegban YCM, Kabran J-P, Koffi FM, Koffi K, Pennetier C. Efficacy of Interceptor® G2, a new long-lasting insecticidal net against wild pyrethroid-resistant Anopheles gambiae ss from Côte d'lvoire: a semi-field trial. Parasite $2018,25$.

65. Clegban C-MY, Camara S, Koffi AA, Alou LPA, Kouame J-PK, Koffi AF, Kouassi PK, Moiroux N, Pennetier C. Evaluation of Yahe® and Panda ${ }^{\circledR} 2.0$ long-lasting insecticidal nets against wild pyrethroid-resistant Anopheles gambiae sl from Côte d'Ivoire: an experimental hut trial. Parasites Vectors. 2021;14:1-11.

66. Kweka EJ, Himeidan YE, Mahande AM, Mwang'onde BJ, Msangi S, Mahande MJ, Mazigo HD, Nyindo M. Durability associated efficacy of long-lasting insecticidal nets after five years of household use. Parasites vectors. 2011;4:1-5.

67. Kweka EJ, Lyaruu LJ, Mahande AM. Efficacy of PermaNet ${ }^{\circledR} 3.0$ and PermaNet ${ }^{\circledR} 2.0$ nets against laboratory-reared and wild Anopheles gambiae sensu lato populations in northern Tanzania. Infectious diseases of poverty. 2017;6:1-10.

68. Kweka EJ, Tungu PK, Mahande AM, Mazigo HD, Sayumwe S, Msangi S, Lyaruu L, Waweru J, Kisinza W, Wangai J. Bio-efficacy and wash resistance of MAGNet long-lasting insecticidal net against wild populations of Anopheles funestus in experimental huts in Muheza, Tanzania. Malar J. 2019;18:1-8.

69. Mahande AM, Msangi S, Lyaruu LJ, Kweka EJ. Bio-efficacy of DuraNet® long-lasting insecticidal nets against wild populations of Anopheles arabiensis in experimental huts. Tropical medicine health. 2018;46:1-8.

70. Musa JJ, Moore SJ, Moore J, Mbuba E, Mbeyela E, Kobe D, Swai JK, Odufuwa OG. Long-lasting insecticidal nets retain bio-efficacy after 5 years of storage: implications for malaria control programmes. Malar J. 2020;19:1-12.

71. Ngufor C, Agbevo A, Fagbohoun J, Fongnikin A, Rowland M. Efficacy of Royal Guard, a new alpha-cypermethrin and pyriproxyfen treated mosquito net, against pyrethroid-resistant malaria vectors. Scientific reports. 2020;10:1-15.

72. Pennetier C, Bouraima A, Chandre F, Piameu M, Etang J, Rossignol M, Sidick I, Zogo B, Lacroix M-N, Yadav R. Efficacy of Olyset ${ }^{\circledR}$ Plus, a new long-lasting insecticidal net incorporating permethrin and piperonil-butoxide against multi-resistant malaria vectors. Plos one. 2013;8:e75134.

73. Sood RD, Mittal P, Kapoor N, Razdan R, Dash A. Wash resistance and efficacy of Olyset® net and Permanet ${ }^{\circledR} 2.0$ against Anopheles stephensi in India. J Am Mosq Control Assoc. 2011;27:423-8.

74. WHO. Report of the seventh WHOPES working group meeting: WHO. World Health Organization; 2004.

75. WHO. Report of the twelfth WHOPES working group meeting, WHO/HQ, Geneva, 8-11 December 2008: review of bioflash GR, permanet 2.0, permanet 3.0, permanet 2.5, lambda-cyhalothrin LN. 2009.

76. WHO. Report of the fourteenth [14th] WHOPES working group meeting: WHO/HQ, Geneva. World Health Organization; 2011.

77. WHO. Report of the fifteenth WHOPES working group meeting: WHO. 2012.

78. WHO. Report of the sixteenth WHOPES working group meeting: WHO/HQ, Geneva. 2013.

79. WHO. Prequalification Team Vector Control Decision Document Royal Sentry 2.0 (Long Lasting Mosquito Net Treated with Alphacypermethrin). World Health Organization; 2019.

80. WHO. Decision Document Tsara Soft (Deltamethrin Insecticide Treated Net). Prequalification Unit - Vector Control Products Assessment (PQT/NCP). World Health Organization; 2020.

\section{Figures}




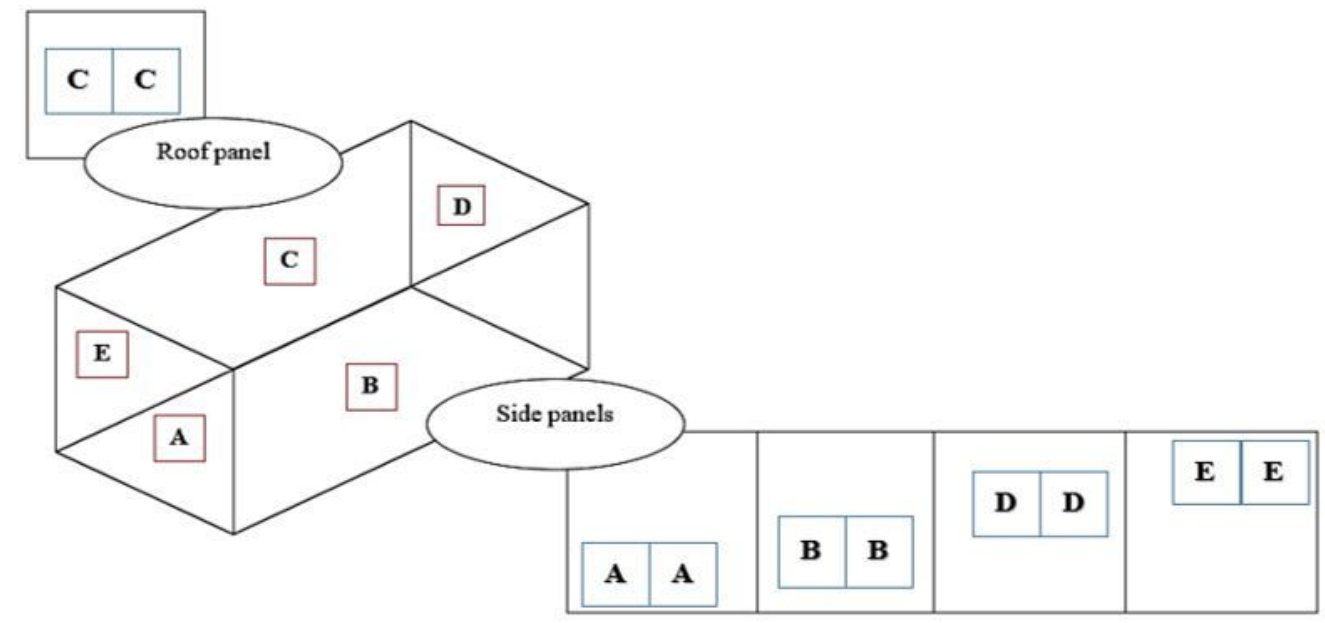

Figure 1

Rectangular whole net with five sides; net piece samples were cut from bottom side (A), middle side A (B), roof (C), middle side B (D) and top side (E).

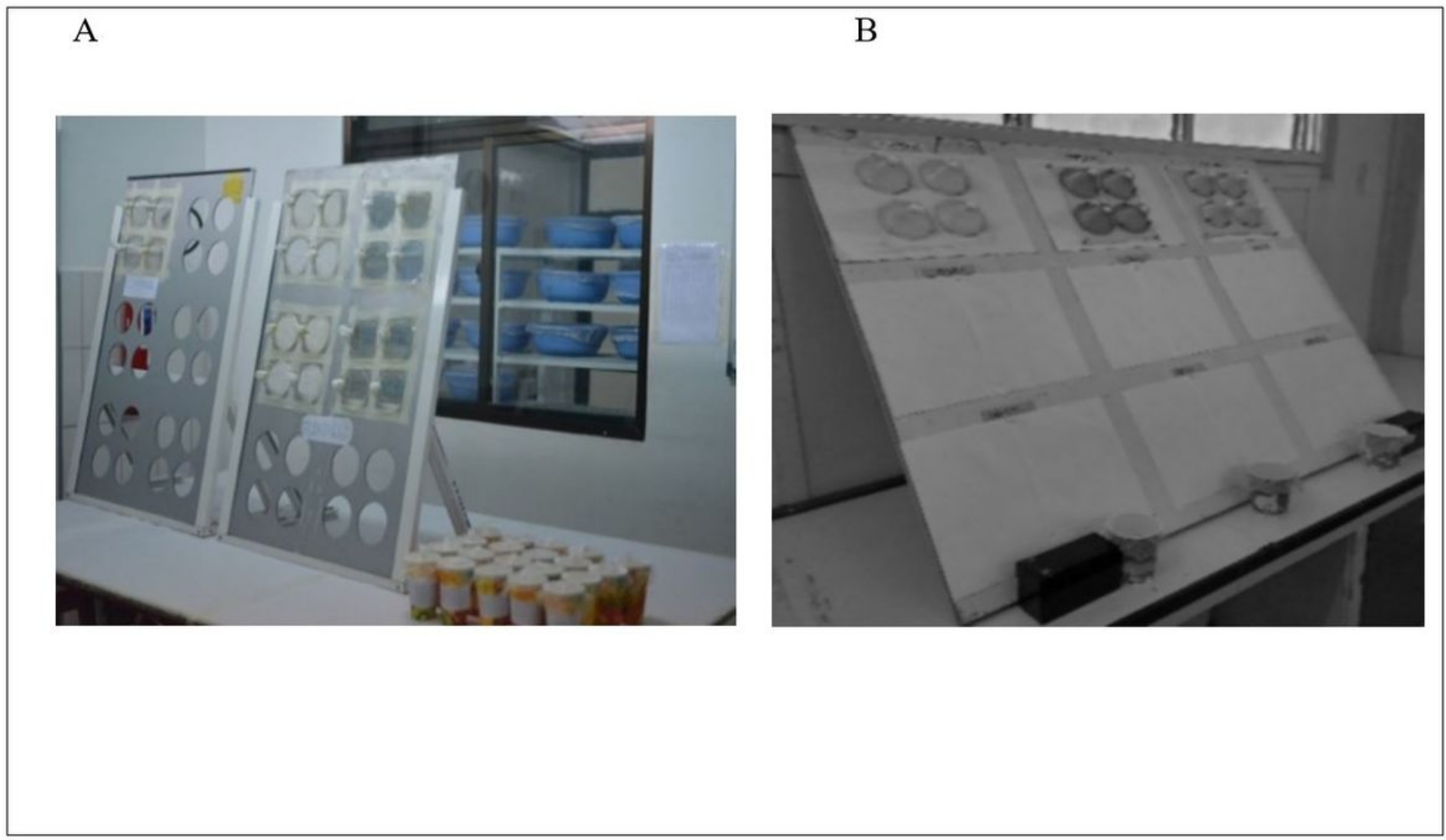


Figure 2

WHO cones fixed on plastic cone board held at $60^{\circ}$ in IHI (A) and at $45^{\circ}$ in PNGIMR (B)

A

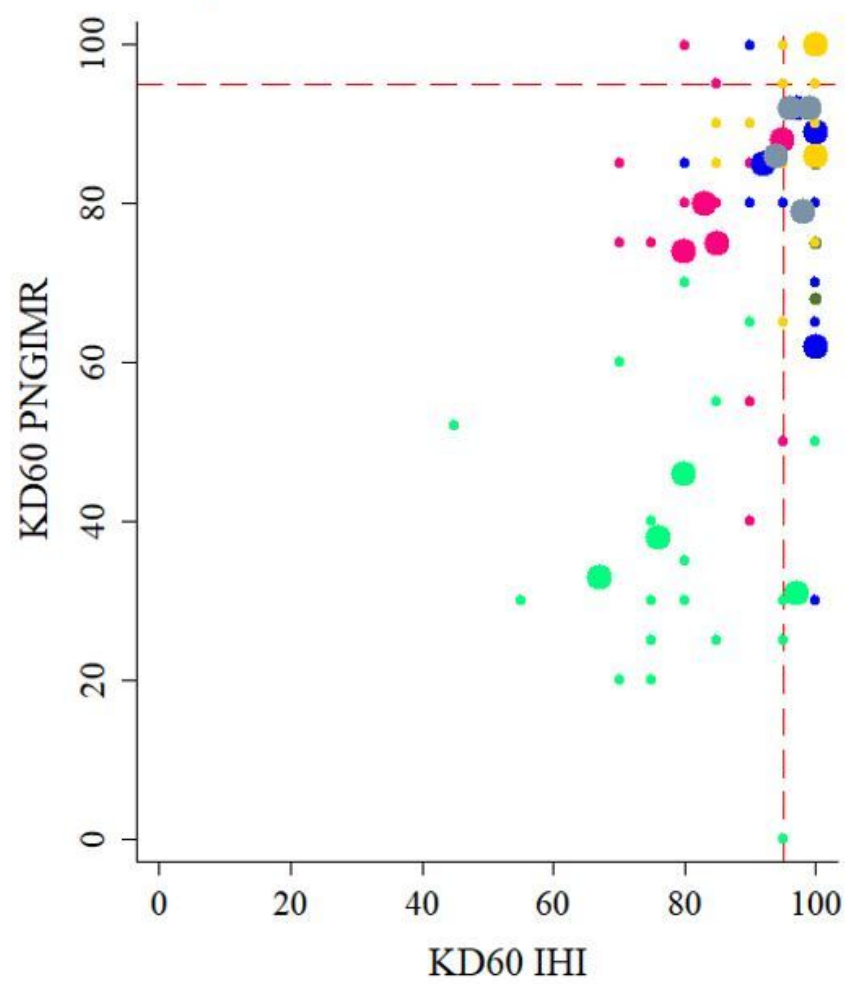

B

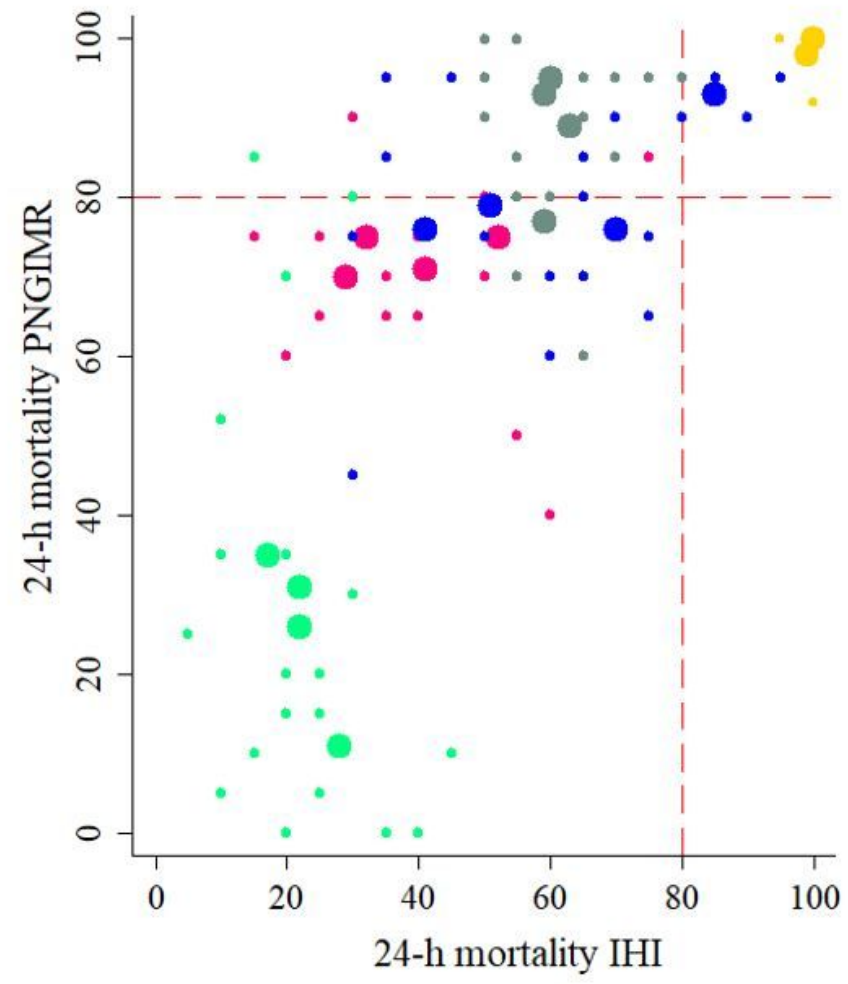

\section{Figure 3}

Correlation of cone bioassay tests results between IHI and PNGIMR testing facilities. Dash line is the WHO threshold $95 \%$ KD60 (A) and $80 \%$ M24 (B). Large dots represent averages per sampled nets (4 per net type) and small dots represent all subsamples (5 per net). 


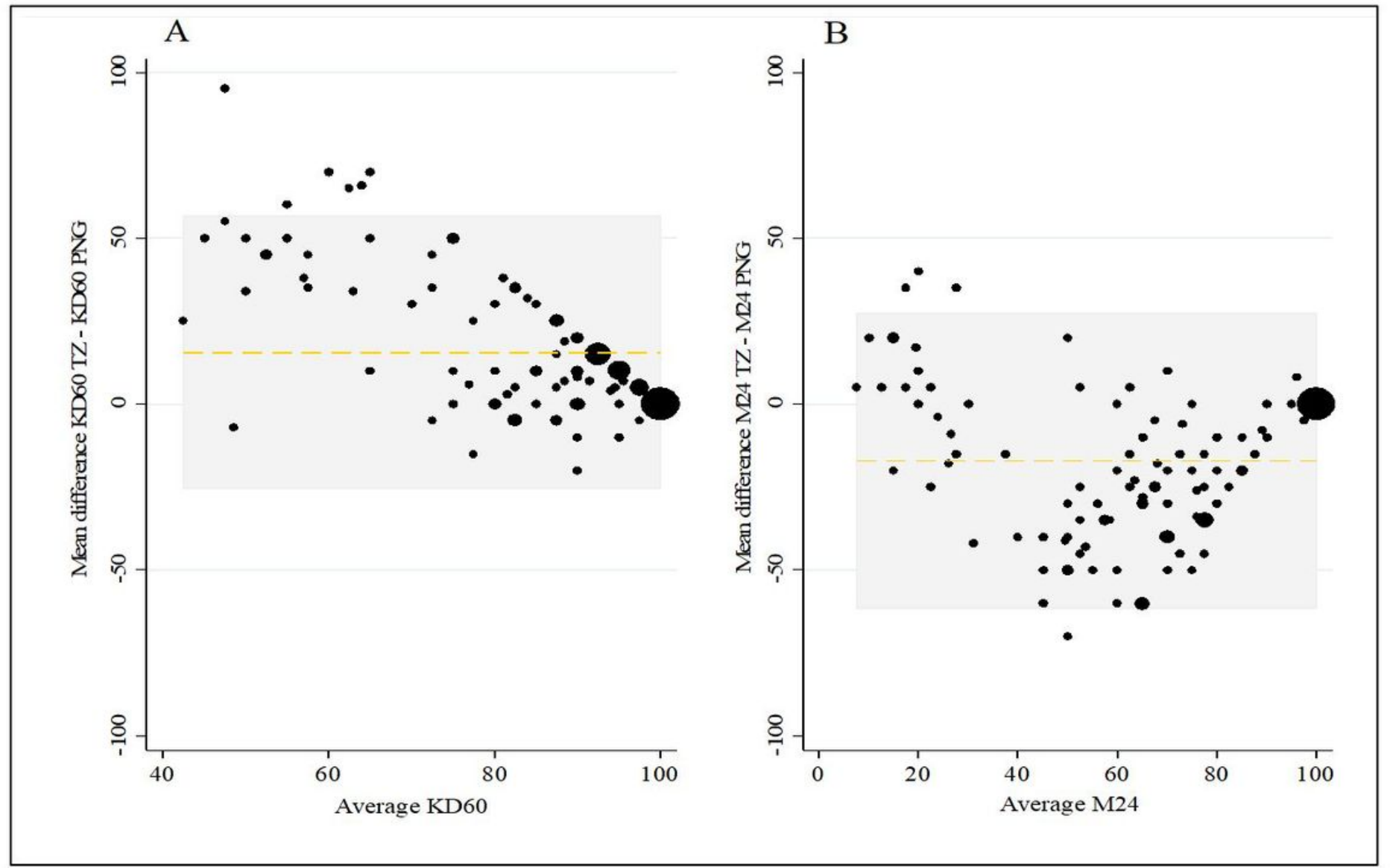

\section{Figure 4}

Bland-Altman Plot showing the mean difference (y axis) plotted against the average value from both sites ( $\mathrm{x}$ ) of mosquitoes' knockdown (A) and mortality at 24 hours (B). For KD60 Mean difference (limits of agreement) knockdown 15.5 (-25.4 to 56.5) and M24 -17.0 (-61.4 to 27.3). At lower mean values of knockdown, the agreement between the two testing facilities was lower than at higher mean values of knockdown but there was a consistent difference in mean difference in M24 measures at each testing facility. 
A

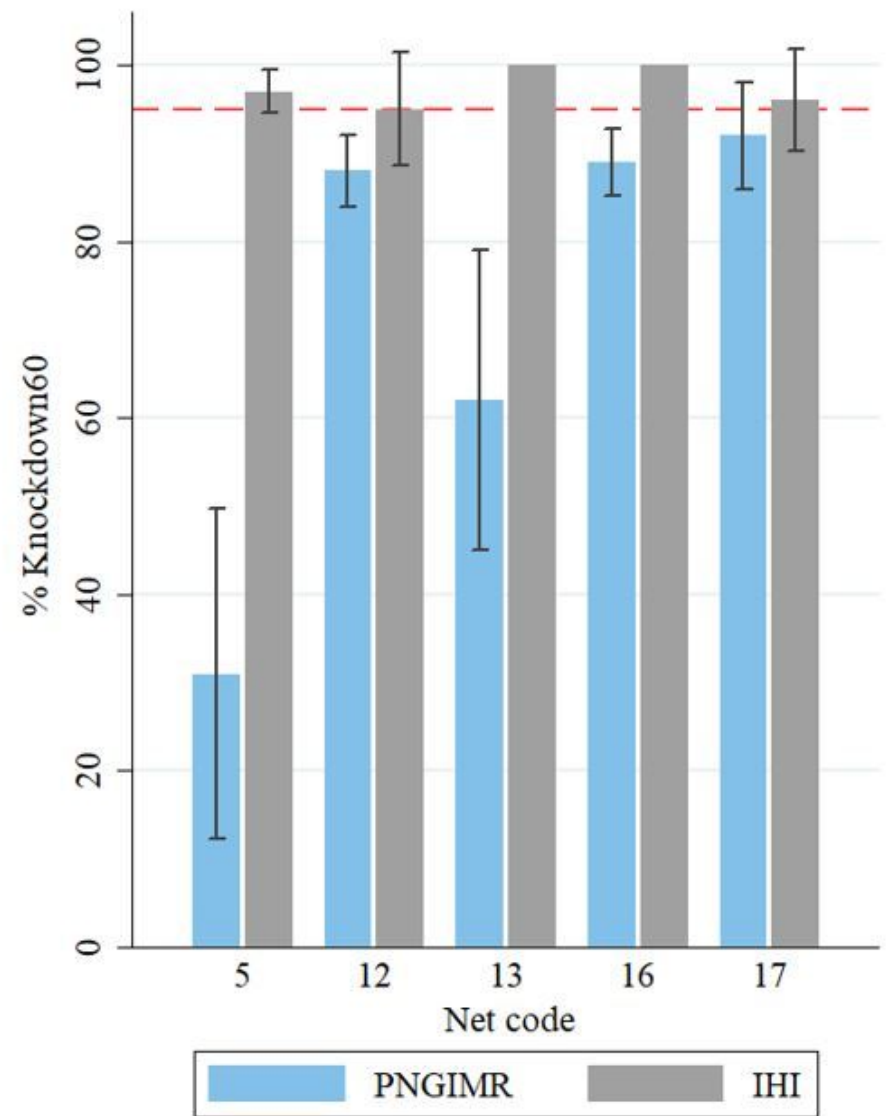

B

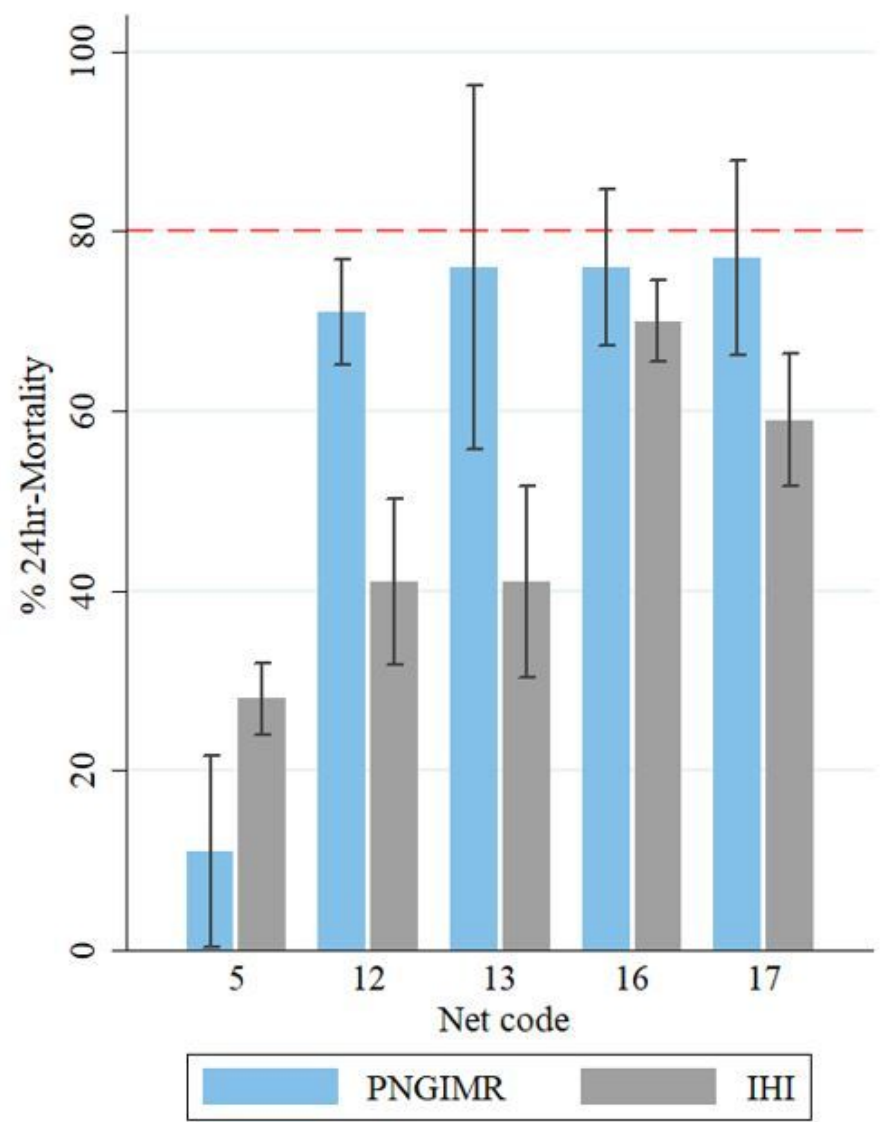

Figure 5

Bioefficacy of the five Insecticide Treated Nets that demonstrated discordant results between IHI and PNGIMR. Each ITN passed efficacy criteria in IHI using the KD optimal bioefficacy criterion of $95 \% \mathrm{KD}(\mathrm{A})$ but did not reach the optimal bioefficacy criterion of $80 \% 24$ hour mortality (B). Three of the nets showed mean 24 hour mortality close to $80 \%$ at PNGIMR with confidence intervals that overlapped the optimal bioefficacy threshold of $80 \%$ mortality (B).

Dash line is the WHO threshold 95\% KD60 and $80 \%$ M24. 
A

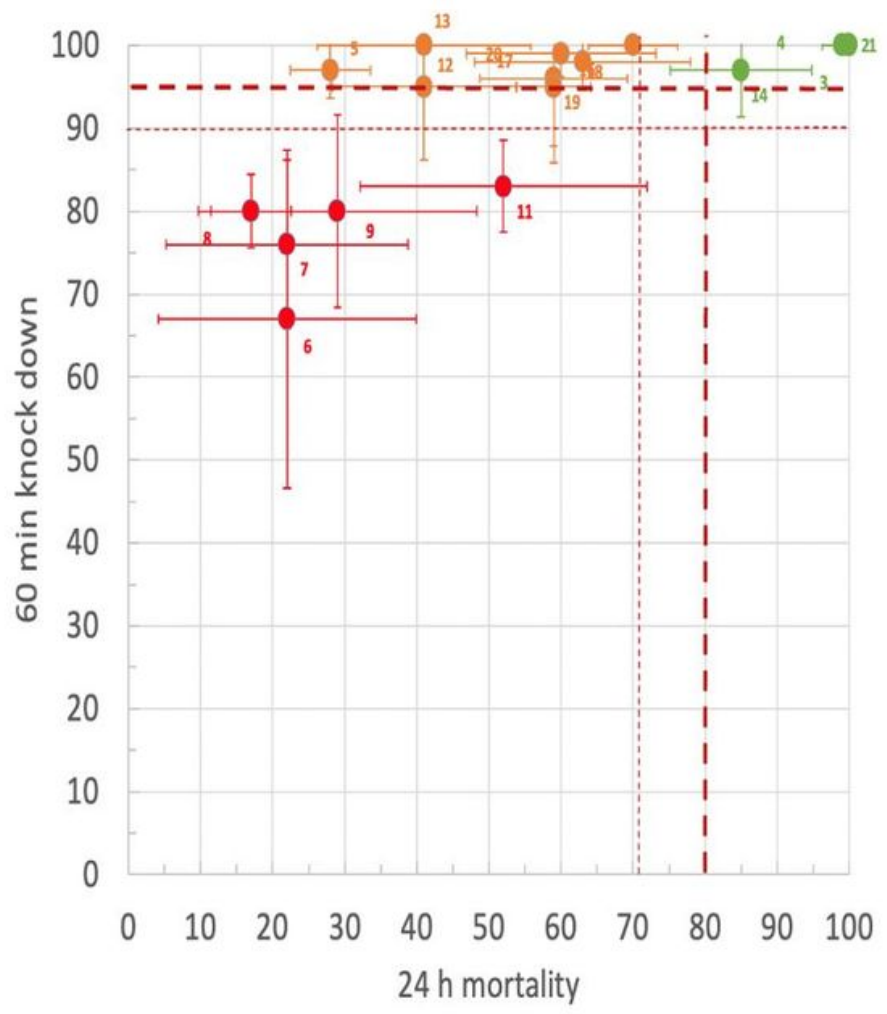

B

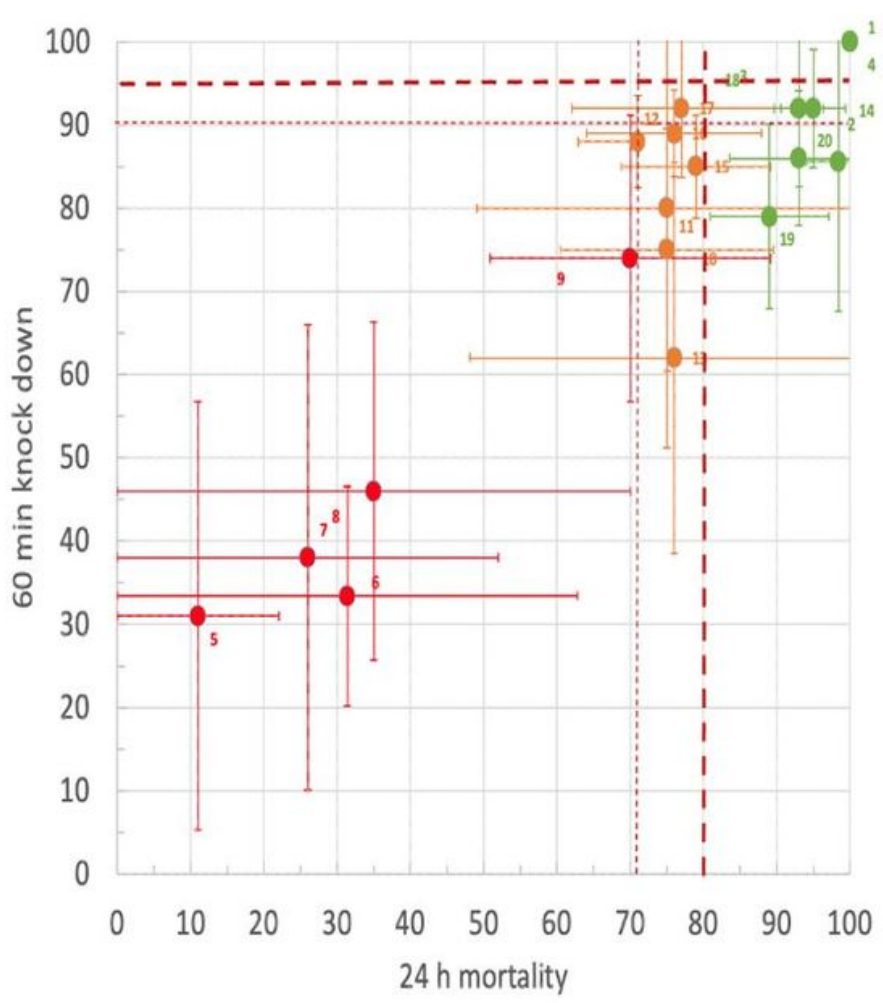

\section{Figure 6}

Show the trends of ITNs passing on mortality (green) or failing (red) WHO cone bioassay criteria, and borderline nets (amber) with variability in the cone bioassay results at IHI (A) and at PNGIMR (B). A thick dash line is the WHO threshold $95 \% 60$-minutes knockdown and $80 \% 24$-hours mortality and a thinner dash line indicate these inherent 95\% (lower) confidence level of the threshold vary between $89 \%$ and $98 \%$ KD60 and $71 \%$ and $87 \%$ M24. Borderline nets are those that pass on KD60 only at IHI or above the $71 \%$ mortality threshold at PNGIMR. Nets for which there is a disparity are nets 5 and 12 that passed at IHI but not PNGIMR. 
A

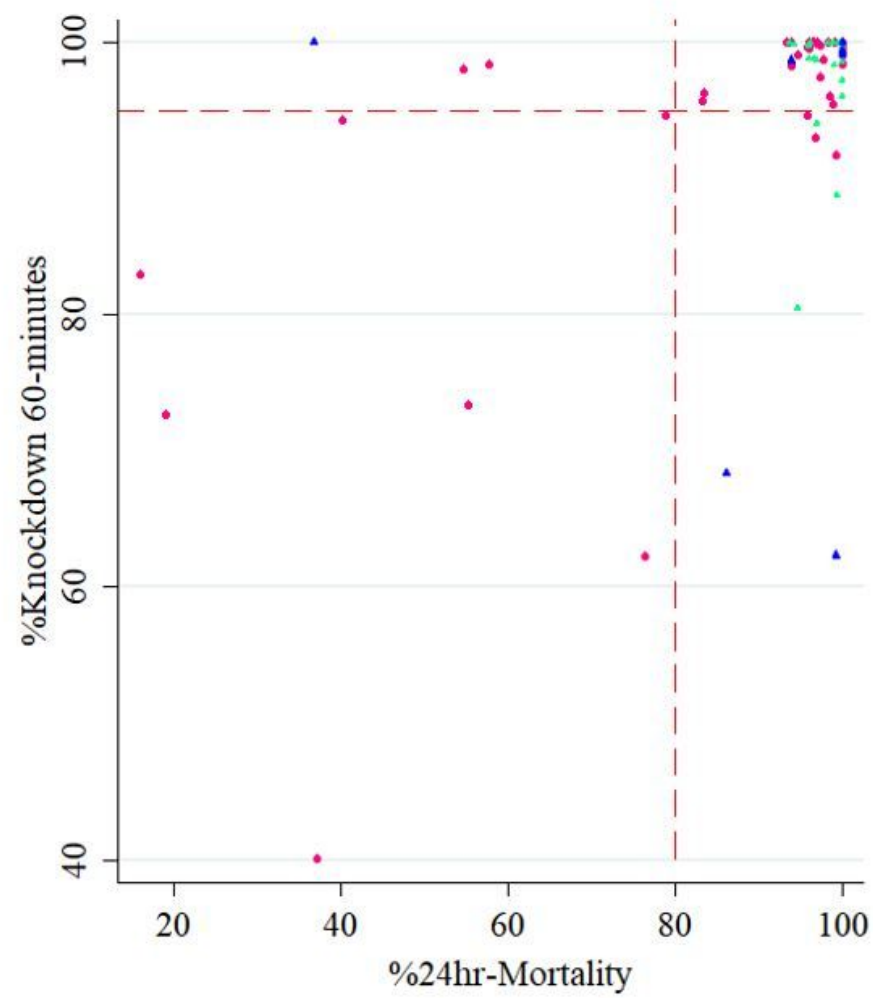

- Deltamethrin Alpha-cypermethrin

- Permethrin
$\mathrm{B}$

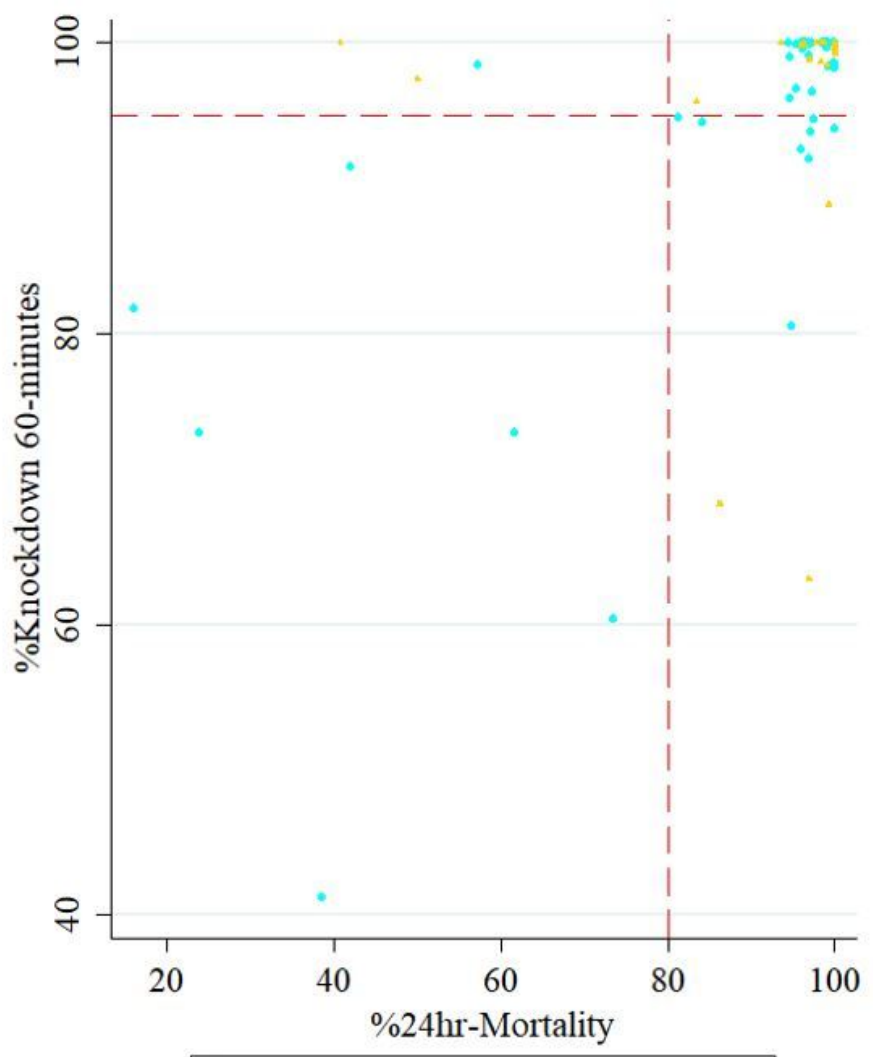

Impregnation Incorporation

Figure 7

Relationship between knockdown and mortality of Anopheles mosquitoes on cone bioassay in ITNs with pyrethroid insecticides (deltamethrin, alpha-cypermethrin and permethrin) (A) and relationship between knockdown and mortality of Anopheles mosquitoes on cone bioassay in ITN production technology (B). Dash line is the WHO threshold 95\% knockdown 60 minutes and $80 \% 24$-hours mortality.

\section{Supplementary Files}

This is a list of supplementary files associated with this preprint. Click to download.

- AdditionalfileNetdetails.xlsx 